Numerical Simulation of Vortex-Induced Vibration of a Vertical Riser in Uniform and Linearly Sheared Currents

Enhao Wang and Qing Xiao

NOTICE: this is the author's version of a work that was accepted for publication in the Journal of Ocean Engineering. Changes resulting from the publishing process, such as final peer review, editing, corrections, structural formatting, and other quality control mechanisms may not be reflected in this document. Changes may have been made to this work since it was submitted for publication. This manuscript was accepted for publishing on 02 June 2016. 


\title{
Numerical Simulation of Vortex-Induced Vibration of a Vertical Riser in Uniform and Linearly Sheared Currents
}

\author{
Enhao Wang and Qing Xiao* \\ Department of Naval Architecture, Ocean and Marine Engineering, University of Strathclyde, Henry Dyer \\ Building, Glasgow G4 OLZ, Scotland, UK
}

\begin{abstract}
This paper presents a numerical study on vortex-induced vibration (VIV) of a vertical riser subject to uniform and linearly sheared currents. The model vertical riser tested at the MARINTEK by ExxonMobil is considered. The predicted numerical results are in good agreement with the experimental data. It is found that the dominant mode numbers, the maximum root mean square amplitudes, the dominant frequencies and the fatigue damage indices increase with the flow velocity. A standing wave response is observed for the singlemode in-line (IL) and cross-flow (CF) vibrations. Dual resonance is found to occur at most of the locations along the riser. At some locations along the riser, a third harmonic frequency component is observed in the $\mathrm{CF}$ response and a frequency component at the $\mathrm{CF}$ response frequency is found in the IL response apart from the frequency component at twice the CF response frequency. The majority of the vortex shedding shows a clear $2 \mathrm{~S}$ pattern, whereas a $2 \mathrm{P}$ mode is observed near the position where the maximum vibration amplitude appears. The higher IL fatigue damage in the present study emphasises the importance of the IL fatigue damage especially in the design of low flow velocity or low mode number applications.
\end{abstract}

Keywords: Vortex-induced vibration (VIV); Riser; Fluid-structure interaction (FSI); Computational fluid dynamics (CFD);

\footnotetext{
* Corresponding author. Tel: +44 1415484779.

Email address: qing.xiao@strath.ac.uk (Q. Xiao)
} 


\begin{tabular}{|c|c|}
\hline Nomenclature & \\
\hline$A_{x r m s} / D, A_{y r m s} / D$ & $\begin{array}{l}\text { Dimensionless in-line and cross-flow root mean square } \\
\text { amplitudes }\end{array}$ \\
\hline$A_{x r m s}^{\max } / D, A_{y r m s}^{\max } / D$ & Dimensionless maximum root mean square amplitudes \\
\hline$c$ & Structural damping \\
\hline$D, D_{o}$ & Riser outer diameter \\
\hline$E$ & Young's modulus \\
\hline$f_{n}$ & Natural frequency of the oscillating mode \\
\hline$f_{n, \text { beam }}$ & $n^{\text {th }}$ eigenfrequency for a nontensioned beam \\
\hline$f_{n, \text { string }}$ & $n^{\text {th }}$ eigenfrequency for a tensioned string \\
\hline$f_{o x}, f_{o y}$ & In-line and cross-flow oscillation frequencies \\
\hline$f_{z, \varepsilon}$ & Zero-crossing frequency of the bending strain \\
\hline$I$ & Moment of inertia of the beam \\
\hline$L$ & Length of the riser \\
\hline$m$ & Mass per unit length of the riser \\
\hline$m^{*}=m /\left(\rho \pi D^{2} / 4\right)$ & Mass ratio \\
\hline$n$ & Mode number \\
\hline $\operatorname{Re}=V D / v$ & Reynolds number \\
\hline$T$ & Top tension \\
\hline$t$ & Instant time \\
\hline$t_{w}$ & Riser wall thickness \\
\hline$V$ & Uniform flow velocity \\
\hline$V_{\max }, V_{\min }$ & Maximum and minimum velocity \\
\hline$V_{\text {profile }}$ & Velocity profile \\
\hline$x$ & In-line displacement \\
\hline$x_{\text {mean }}$ & Mean in-line displacement \\
\hline$y$ & Cross-flow displacement \\
\hline$\varepsilon$ & Root mean square strain \\
\hline$\rho$ & Fluid density \\
\hline$v$ & Kinematic viscosity of the fluid \\
\hline
\end{tabular}




\section{Introduction}

Vortex-induced vibration (VIV) often causes the fatigue of offshore slender structures, such as risers, mooring lines and pipelines. Detailed understanding of this fluid-structure interaction (FSI) phenomenon and an efficient prediction of such self-excited and selfsustained oscillations are required for the reliable estimation of the fatigue damage and the development of VIV suppression techniques (Bourguet et al., 2011a, 2013).

Over the past few decades, VIV has been extensively studied. One may refer to the comprehensive reviews by Sarpkaya (1979), Bearman (1984), Williamson and Govardhan (2004), Gabbai and Benaroya (2005), Bearman (2011) and more recently by Wu et al. (2012).

As riser pipes often possess a length-to-diameter ratio $(L / D)$ of the order of $10^{3}$ (Chaplin et al., 2005), many experiments have been carried out on deepwater risers with large $L / D$ (Tognarelli et al., 2004; Chaplin et al., 2005; Trim et al., 2005; Lie and Kaasen, 2006; Vandiver et al., 2006; Tognarelli et al., 2008; Vandiver et al., 2009; Huang et al., 2011b; Gu et al., 2013; Gao et al., 2015). These experiments investigated flexible riser VIV responses under different flow conditions and some also assessed the effectiveness of VIV suppression techniques, such as using helical strakes. Better insights into some important VIV aspects (i.e., response amplitude, dominant mode, dominant frequency and fatigue damage etc.) were obtained from these experiments, and thus provided some good benchmarks for verifying numerical prediction models.

Apart from the various experimental investigations, there have been a number of computational fluid dynamics (CFD) studies on VIV of flexible cylinders.

Willden and Graham (2001) used a quasi-three-dimensional (Q3D) method to simulate the transverse vibration of an $L / D=100$ cylinder subject to a sheared inflow at low Reynolds numbers. A high tension was applied to the cylinder so that the fundamental mode would be excited. A maximum amplitude of $0.36 D$ was found at $L / D=44$ which was slightly below the midpoint of the cylinder span. The results also showed that the majority of the shedding frequencies along the cylinder were modified towards the natural frequency and a significant spanwise correlation was observed. 
Meneghini et al. (2004) and Yamamoto et al. (2004) presented the numerical simulations of long marine risers with $L / D$ up to 4600 with Q3D discrete vortex method (DVM). In their simulations, the riser tended to select a vibration mode which could keep the reduced velocity $V_{r}=V / f_{n} D$ in the range of $4 \leq V_{r} \leq 7$ where the energy was transferred from the fluid to the structure. Visualisations of the wake indicated a hybrid mode of vortex shedding along the span with a $2 \mathrm{~S}$ mode being found in regions of small amplitudes, changing to a $2 \mathrm{P}$ mode in regions of larger amplitudes.

The simulations described above were based on Q3D method with several two-dimensional (2D) strips over the length of the riser. However, Q3D simulations have many shortcomings, e.g., three-dimensional (3D) vortex structures cannot be treated correctly and straked risers and variations in the angle of attack cannot be studied directly. Therefore, a series of fully 3D numerical simulations emerged.

Newman and Karniadakis (1997) simulated VIV of an infinitely long flexible cable at $\mathrm{Re}=$ 100 and $\operatorname{Re}=200$ with a spectral/hp element method. Both the standing wave and travelling wave responses were realized. It was found that an interwoven pattern of vorticity was associated with a standing wave cable response while oblique vortex shedding was produced by a travelling wave cable response. A mixed standing wave/travelling wave response together with chevron-like vortex shedding was found to be related to a sheared inflow.

Evangelinos and Karniadakis (1999) studied VIV of an infinitely long flexible cylinder at Re $=1000$. The structure's bending stiffness was varied to obtain different responses. The authors found that the modulated travelling wave motion of a free-free beam or cable led to a mixed response consisting of oblique and parallel shedding. In the case of structures with pinned endpoints a standing wave response was obtained with lace-like flow structures.

Holmes et al. (2006) and Menter et al. (2006) investigated riser VIV with fully 3D finite element method (FEM) and finite volume method (FVM), respectively. Both of the simulations used relatively coarse meshes with high element aspect ratios and the results were in good agreement with the experimental data by Trim et al. (2005) and Chaplin et al. (2005), respectively. 
Constantinides and Oakley (2008) compared their CFD results with the data obtained in the field experiments by Jhingran and Vandiver (2007). The results were able to match the experimental data. Both the first and third harmonic components were well captured. The authors emphasised the importance of the third harmonic component in fatigue damage analysis due to the fact that it produced strains of the same order of magnitude as the first harmonic component and had a frequency of three times the first harmonic component, which returned roughly three times more fatigue damage.

Huang et al. (2009, 2011a) performed finite-analytic Navier-Stokes (FANS) simulations on three different risers with $L / D$ ranging from 482 to 3350 . The simulation results showed good agreement with the experimental data by Lehn (2003) and Trim et al. (2005) and the numerical results using other commercial software by Holmes et al. (2006). It was observed in their simulations that the VIV of a long riser tended to have more than one dominant modes. The dominant modes could be sensitive to the incoming flow velocity profile and the riser tension. The cross-flow (CF) VIV was influenced by the in-line (IL) deflection. Vortex shedding showed a $2 \mathrm{~S}$ pattern and the CF VIV demonstrated higher harmonic responses.

Bourguet et al. (2011a, b, c, 2012, 2013, 2015) did a series of fundamental studies on VIV of long flexible cylinders. Their research revealed some important flexible cylinder VIV mechanisms, such as the occurrence of lock-in, the orbital trajectories which dominate the wake-body resonance, the phasing mechanisms between the IL and CF VIV and the validity of the independence principle (IP) applied to VIV.

Nevertheless, fully 3D FSI simulations of VIV of a vertical riser subject to various flow conditions are still quite limited. Past studies found that the dominant modes were related to the incoming flow velocity profile (Huang et al., 2011a) and the IL VIV should not be neglected in deepwater riser design (Tognarelli et al., 2004; Xue et al., 2015). However, most of the previous CFD studies on vertical riser VIV focussed on a single flow condition and the importance of the IL fatigue damage, especially at low flow velocities, was not fully addressed. Therefore, in this paper, combined IL and CF VIV of a vertical riser in uniform and linearly sheared currents is studied using a fully 3D CFD approach. A low flow velocity range is specially selected to cover the typical range where the IL fatigue damage is higher than the CF fatigue damage so that the importance of the IL fatigue damage can be addressed. The rest of the paper are organised as follows. The numerical methods are given in Section 2 
and a detailed description of the problem is provided in Section 3. In Section 4, simulation results of VIV of a vertical riser in uniform and linearly sheared currents are presented and in-depth comparisons are made with the experimental data. Finally, the conclusions of this paper are summarised in Section 5.

\section{Numerical methods}

A commercial software package ANSYS MFX multi-field solver has been adopted to solve the FSI problem in this paper. The numerical methods are summarised as follows.

\subsection{Flow model}

The flow field around the riser is modelled by solving the unsteady, incompressible NavierStokes equations in conjunction with the large eddy simulation (LES) wall-adapted local eddy-viscosity (WALE) model (Nicoud and Ducros, 1999). In this study, the Arbitrary Lagrangian-Eulerian (ALE) scheme is applied to deal with the moving boundary of the cylinder. The ALE form of the governing equations in the Cartesian coordinate system is expressed as

$$
\begin{gathered}
\frac{\partial \bar{u}_{i}}{\partial x_{i}}=0 \\
\frac{\partial \bar{u}_{i}}{\partial t}+\left(\bar{u}_{j}-\hat{u}_{j}\right) \frac{\partial \bar{u}_{i}}{\partial x_{j}}=-\frac{1}{\rho} \frac{\partial \bar{p}}{\partial x_{i}}+\frac{\partial}{\partial x_{j}}\left[v\left(\frac{\partial \bar{u}_{i}}{\partial x_{j}}+\frac{\partial \bar{u}_{j}}{\partial x_{i}}\right)\right]-\frac{\partial \tau_{i j}}{\partial x_{j}}
\end{gathered}
$$

where $\left(x_{1}, x_{2}, x_{3}\right)=(x, y, z)$ are the Cartesian coordinates, an overbar denotes that the variable is a filtered variable, $u_{i}$ is the velocity component in the $x_{i}$ direction, $\hat{u}_{i}$ is the grid velocity component in the $x_{i}$ direction, $p$ is the pressure, $t$ is the time, $\rho$ is the fluid density, $v$ is the kinematic viscosity of the fluid and $\tau_{i j}$ is the subgrid-scale stress defined by

$$
\tau_{i j}=\overline{u_{i} u_{j}}-\overline{u_{i}} \bar{u}_{j}
$$

Based on the Boussinesq's approximation

$$
-\left(\tau_{i j}-\frac{\delta_{i j}}{3} \tau_{k k}\right)=2 v_{s g s} \bar{S}_{i j}
$$


where $\delta_{i j}$ is the Kronecker symbol. The isotropic part of the subgrid-scale stresses $\tau_{k k}$ is not modelled, but added to the filtered static pressure. $\bar{S}_{i j}$ is the rate-of-strain tensor for the resolved scale defined by

$$
\bar{S}_{i j}=\frac{1}{2}\left(\frac{\partial \bar{u}_{i}}{\partial x_{j}}+\frac{\partial \bar{u}_{j}}{\partial x_{i}}\right)
$$

The eddy-viscosity is computed by

$$
v_{s g s}=\left(C_{w} \Delta\right)^{2} \frac{\left(S_{i j}^{d} S_{i j}^{d}\right)^{3 / 2}}{\left(\bar{S}_{i j} \bar{S}_{i j}\right)^{5 / 2}+\left(S_{i j}^{d} S_{i j}^{d}\right)^{5 / 4}}
$$

The model constant $C_{w}$ is chosen as 0.325 . The filter-width is taken as the local grid size, i.e., $\Delta=(\Delta x \Delta y \Delta z)^{1 / 3} . S_{i j}^{d}$ denotes the traceless symmetric part of the square of the velocity gradient tensor:

$$
S_{i j}^{d}=\frac{1}{2}\left(\bar{g}_{i j}^{2}+\bar{g}_{j i}^{2}\right)-\frac{1}{3} \delta_{i j} \bar{g}_{k k}^{2}
$$

where $\bar{g}_{i j}^{2}=\bar{g}_{i k} \bar{g}_{k j}, \bar{g}_{i j}=\partial \bar{u}_{i} / \partial x_{j}$. The tensor $S_{i j}^{d}$ can be rewritten in terms of the strain-rate and vorticity tensors:

$$
S_{i j}^{d}=\bar{S}_{i k} \bar{S}_{k j}+\bar{\Omega}_{i k} \bar{\Omega}_{k j}-\frac{1}{3} \delta_{i j}\left(\bar{S}_{m n} \bar{S}_{m n}-\bar{\Omega}_{m n} \bar{\Omega}_{m n}\right)
$$

where the vorticity tensor is given by

$$
\bar{\Omega}_{i j}=\frac{1}{2}\left(\frac{\partial \bar{u}_{i}}{\partial x_{j}}-\frac{\partial \bar{u}_{j}}{\partial x_{i}}\right)
$$

The main advantages of the LES WALE model are the capability of reproducing the laminar to turbulent transition and the design of the model to return the correct wall-asymptotic $y^{+3}$ (ANSYS Inc., 2013).

The governing equations are discretised using an element-based FVM. Rhie-Chow interpolation is used to obtain pressure-velocity coupling on collocated grids. A second-order backward Euler scheme is adopted for the temporal discretisation and a bounded central difference scheme is used as the convection scheme.

\subsection{Structural dynamic model}


According to Huang et al. (2011a), a top tensioned riser can be simplified as a tensioned beam whose lateral motion is described as

$$
\frac{\partial^{2}}{\partial z^{2}}\left[E I \frac{\partial^{2} x_{i}}{\partial z^{2}}\right]-\frac{\partial}{\partial z}\left[T \frac{\partial x_{i}}{\partial z}\right]+m \frac{\partial^{2} x_{i}}{\partial t^{2}}+c \frac{\partial x_{i}}{\partial t}=F_{i}
$$

where $E$ is the Young's modulus, $I$ is the moment of inertia, $T$ is the top tension, $m$ is the mass per unit length, $c$ is the structural damping, $z$ is the undeflected riser axis, $x_{1}$ and $x_{2}$ denote the IL and CF displacements, respectively and $F_{1}$ and $F_{2}$ are the hydrodynamic forces in the IL and CF directions, respectively.

A finite element method is used to discretise Eq. (10) and the governing equation is given by

$$
[M]\{\ddot{q}\}+[C]\{\dot{q}\}+[K]\{q\}=\{F\}
$$

where $\{q\}$ is the nodal displacement vector and a dot denotes differentiation with respect to time. $[M],[C]$ and $[K]$ are the mass, damping and stiffness matrices, respectively. $\{F\}$ is the hydrodynamic force vector. The governing equation is solved using the Hilber-HughesTaylor (HHT) method (Chung and Hulbert, 1993) with the second order accuracy.

\subsection{Mesh deformation}

To accommodate the motion of the riser, the displacement diffusion model (Zhao and Cheng, 2011 ; Zhao et al., 2014) for mesh motion is adopted. The displacements of the mesh points are calculated based on the following equation:

$$
\nabla \cdot\left(\gamma \nabla S_{i}\right)=0
$$

where $S_{i}$ represents the displacements of the nodal points in the $x_{i}$ direction, $\gamma$ is the mesh stiffness. In this study, in order to avoid excessive deformation of the near-wall elements, the parameter $\gamma$ is set to be $\gamma=1 / \forall^{2}$ where $\forall$ is the control volume size.

\subsection{Fluid-structure interaction}

A two-way explicit approach is utilised in the present FSI simulation, i.e., the fluid and solid equations are solved separately and there are no iterations between the fluid and solid fields within one time step. The flow chart of the two-way explicit FSI solution procedures for one time step is shown in Fig. 2. It can be seen that within one time step, the flow equations are 
solved to obtain the forces on the riser. Then the forces are interpolated to the structural mesh using the conservative interpolation and the structural dynamic equation is solved to obtain the quantities of the riser motion. After that, the displacements are interpolated to the fluid mesh with the profile preserving interpolation and the positions of the mesh points are calculated and updated using the displacement diffusion model. The next time step begins with solving the flow equations on the updated mesh.

\section{Description of the problem}

\subsection{Simulation parameters}

In the present study, two types of flow conditions are considered for VIV of a vertical riser, i.e., uniform flow and linearly sheared flow. The model vertical riser tested at the MARINTEK by ExxonMobil (Lehn, 2003) is considered. The main parameters of the model riser are summarised in Table 1 . The model riser has a length-to-diameter ratio $L / D=481.5$ and a mass ratio $m^{*}=2.23$. The structural damping in this study is set to be zero. The physical configuration of a vertical riser subject to VIV is displayed in Fig. 1 (a). The flow direction is parallel to the global $x$-axis. A top tension $T=817 \mathrm{~N}$ is applied to the top end of the riser. The riser is pinned at both ends and it is free to move in the IL $(x)$ and CF $(y)$ directions. Simulations are performed for four different test cases in the experiment, namely \# 1103, \# 1105, \# 1201 and \# 1205. The incoming flow velocity and other parameters of the four cases are summarised in Table 2 and the corresponding velocity profiles are displayed in Fig. 1 (b). Detailed descriptions of the flow velocity profiles of the four cases are given as follows.

The case \# 1103 and the case \# 1105 have uniform velocity profiles with $V=0.2 \mathrm{~m} / \mathrm{s}$ and $0.42 \mathrm{~m} / \mathrm{s}$, respectively. Whereas the currents in the case \# 1201 and the case \# 1205 are linearly sheared with the maximum velocities at the bottom end of the riser $(z=0)$ being $V_{\max }$ $=0.2 \mathrm{~m} / \mathrm{s}$ and $V_{\max }=0.42 \mathrm{~m} / \mathrm{s}$, respectively. In both cases, the minimum velocity at the top end of the riser $(z=L) V_{\min }=0.14 V_{\max }$.

To estimate the eigenfrequencies for a vertical riser, it could be simplified as a tensioned beam with moment-free supports at both ends (Lie and Kaasen, 2006). The $n^{\text {th }}$ 
eigenfrequency for the tensioned beam, $f_{n}$, t-beam can be expressed in terms of the eigenfrequencies for a tensioned string and a nontensioned beam (Weaver et al., 1974)

$$
f_{n, t \text {-beam }}=\sqrt{f_{n, \text { string }}^{2}+f_{n, \text { beam }}^{2}}
$$

where $n$ is the mode number, $f_{n, \text { string }}=\frac{n}{2} \sqrt{\frac{T}{m L^{2}}}$ and $f_{n, \text { beam }}=\frac{n^{2} \pi}{2} \sqrt{\frac{E I}{m L^{4}}}$ are the eigenfrequencies for a tensioned string without bending stiffness and a nontensioned beam of equal length $L$ and mass per unit length $m$.

The eigenfrequencies of the foremost eight modes are calculated with Eq. (13) along with a modal analysis carried out using ANSYS software. The results are tabulated in Table 3. The eigenfrequencies from the modal analysis agree well with the corresponding theoretical values with all errors less than $0.1 \%$.

\subsection{Fluid domain and boundary conditions}

Fig. 3 (a) shows the computational domain for the CFD simulation of VIV of a vertical riser. The origin of the Cartesian coordinate system is located at the centre of the bottom end of the riser. The length of the domain is $40 \mathrm{D}$ with the riser being located at $10 \mathrm{D}$ downstream the inlet boundary. The width of the domain in the transverse direction ( $y$-direction) is $20 D$ and the length of the riser is $481.5 \mathrm{D}$. The computational mesh in the $x y$-plane and a zoomed-in view of the mesh around the cylinder are shown in Fig. 3 (b) and Fig. 3 (c), respectively. There are 180 nodes along the circumference of the riser and the minimum mesh size next to the riser surface in the radial direction is $0.001 D$. The non-dimensional mesh size next to the riser surface is found to be $y^{+}<1$, where $y^{+}$is defined as $y^{+}=u_{f} y / v$ with $u_{f}$ being the friction velocity and $y$ being the distance to the nearest wall. The riser starts with a straight configuration (see Fig. 3 (d)) and it deflects towards the current downstream after it is exposed to the different current profiles until its internal restoring force is sufficiently large to overcome the drag forces as shown in Fig. 3 (e). The boundary conditions for the governing equations are as follows. The surface of the cylinder is assumed to be smooth, where no-slip boundary condition is employed. Apart from the no-slip boundary condition, the cylinder surface is also regarded as a fluid-solid interface where the coupling data, i.e., forces and displacements are transferred. The inlet velocity boundary conditions are set to be the same as the freestream velocity. At the outflow boundary, the gradients of the fluid velocity in the 
streamwise direction are set to zero and the pressure at the outflow boundary is given a reference value of zero. On the two spanwise and the two transverse boundaries, the velocity in the direction normal to the boundary is zero.

\subsection{Solid domain and boundary conditions}

Fig. 4 shows the computational mesh for the finite element analysis (FEA) of the present FSI simulation. The scale of the FEA model has been modified in order to clearly view the deflection of the model. Fig. 4 (a) is the initial FEA mesh without riser deflection and Fig. 4 (b) is the FEA mesh with riser deflection. A 3D 20-node solid element SOLID186 which exhibits quadratic displacement behaviour is used for the discretisation of the finite element model. The spanwise direction of the riser finite element model is discretised using 250 segments which is a typical resolution for riser global dynamic analysis (Huang et al., 2009). A top tension $T=817 \mathrm{~N}$ is applied to the top end of the riser. Both the top and bottom ends of the riser are pinned with zero rotational stiffness. The outer surface of the FEA model is also set to be a fluid-solid interface for data transfer.

\subsection{Mesh dependence study}

A multiblock structured mesh is used in the present CFD simulation. The meshing strategy is that a fine mesh is used in the $x y$-plane and a relatively coarse mesh is used in the spanwise direction. The reason why a relatively coarse mesh can be used in the spanwise direction is that the vibration of the riser increases the spanwise correlation of the wake (Blevins, 1977). In other words, the three-dimensionality of the wake reduces as a consequence of the motion of the riser. Therefore, it is possible to obtain reasonable results with relatively coarse mesh in the spanwise direction at the cost of sacrificing the resolution of small scale axial flow features. In order to ensure that the numerical results are independent of the grid size, a mesh dependence test is carried out. Three different meshes are used to simulate the case \# 1105 and the results are compared with the experimental data by Lehn (2003) and the numerical results of Huang et al. (2011a). Table 4 shows the mesh characteristics, the maximum IL root mean square $(\mathrm{rms})$ amplitude $\left(A_{x r m s}^{\max } / D\right)$ and the maximum CF rms amplitude $\left(A_{y r m s}^{\max } / D\right)$ computed using the three mesh systems. Comparing with the experimental data, $A_{x r m s}^{\max } / D$ is slightly underpredicted while $A_{y r m s}^{\max } / D$ is slightly overpredicted using the three meshes. The 
maximum difference of $5.93 \%$ occurs in the value of $A_{x r m s}^{\max } / D$ between Mesh 1 and Mesh 2 whereas the difference between Mesh 2 and Mesh 3 reduces to only 2.4\%. In the meanwhile, the differences in the value of $A_{y r m s}^{\max } / D$ between Mesh 1 and Mesh 2 and between Mesh 2 and Mesh 3 are $3.07 \%$ and 2.24\%, respectively. It can be concluded that the difference in the results predicted by Mesh 2 and Mesh 3 is within the acceptable range. The variation of rms amplitudes along the riser span in the IL and CF directions is displayed in Fig. 5. The IL and CF rms amplitudes and vibration modes predicted by the three meshes are quite similar and the maximum CF rms amplitudes predicted by Mesh 2 and Mesh 3 are in agreement with the numerical results of Huang et al. (2011a) with the CF responses predicted by the two meshes showing better comparison with the experimental data in the upper part of the riser $(z / L>0.6)$ than the results of Huang et al. (2011a). Based on the discussions above and also taking into account the computational efforts, the simulations in this paper are conducted with Mesh 2. As the present FEA mesh is able to predict the riser response with reasonable accuracy, the FEA mesh dependence study is not discussed in this paper.

\section{Results and discussions}

Numerical simulations are performed for VIV of a vertical riser in uniform and linearly sheared currents. The velocity profiles considered are uniform velocities $V=0.2 \mathrm{~m} / \mathrm{s}$ and 0.42 $\mathrm{m} / \mathrm{s}$ and linearly sheared velocity profiles which can be described as $V_{\text {profile }}=(1-$ $0.86 z / L) V_{\max }$ where $V_{\max }=0.2 \mathrm{~m} / \mathrm{s}$ and $0.42 \mathrm{~m} / \mathrm{s}$. Both the IL and CF VIV results are compared with the experimental data by Lehn (2003).

\subsection{Riser dynamic responses}

Fig. 6 shows the comparison of the envelopes of the IL and CF displacements between the present simulation and the experiment. It is clear that the present results are in good agreement with the experimental data in terms of the dominant modes. In the case \# 1103, the IL and CF vibrations are dominated by the second mode and the first mode, respectively. When the uniform flow velocity increases to $V=0.42 \mathrm{~m} / \mathrm{s}$, the dominant modes in the IL and $\mathrm{CF}$ directions change to the third mode and the second mode, respectively. 
Similar to the uniform flow conditions, the dominant modes in the IL and CF directions in the two linearly sheared flow cases also change with the maximum flow velocity. In the present simulation, the variation of the dominant modes in the two linearly sheared flow cases is the same as that in the two uniform flow cases, i.e., the second mode and the first mode in the IL and CF directions for $V_{\max }=0.2 \mathrm{~m} / \mathrm{s}$ transfer into the third mode and the second mode when $V_{\max }$ increases to $0.42 \mathrm{~m} / \mathrm{s}$.

One discrepancy is observed in the IL dominant mode in the case \# 1201. The present numerical simulation predicts a second mode, whereas the dominant mode is the third mode in the experiment. We believe that the present numerical results are more reasonable and the reasons behind are explained as follows.

As an approximation, the IL response frequency may be estimated to be twice the CF response frequency. This implies that the IL mode number is twice the CF mode number for a tensioned string, whereas for a nontensioned beam it is lower, due to the quadratic relationship between $n$ and frequency (Lie and Kaasen, 2006). According to Lehn (2003), the natural frequency of the riser is dominated by tension if $T \geq 4 \pi^{2} n^{2} E I / L^{2}$. For the case \# 1201 we discussed herein, the tension of the riser $T=817 \mathrm{~N}$ is larger than $4 \pi^{2} n^{2} E I / L^{2}$ for $n=1,2$, and 3, therefore the natural frequencies of the first three modes are dominated by tension. It is thus reasonable to expect that the behaviour of the riser would be similar to a tensioned string, whose IL mode number should be twice the CF mode number when the riser vibrates in low mode numbers $(n \leq 3)$. As both the experiment and the present simulated CF dominant modes exhibit the first mode, the expected dominant mode in the IL direction should be the second mode.

The IL and CF motion evolution responses along the riser predicted by the present FSI simulation are analyzed in an effort to understand the riser dynamics. As is shown in Fig. 7, the variation of the dominant mode shapes with the flow conditions agrees with the observations from Fig. 6. Under the uniform flow condition, the IL dominant mode changes from the second to the third mode and the CF dominant mode shifts from the first mode to the second mode when $V$ increases from $0.2 \mathrm{~m} / \mathrm{s}$ to $0.42 \mathrm{~m} / \mathrm{s}$. The variation of the dominant modes in the two linearly sheared flow cases is the same as that in the two uniform flow cases when $V_{\max }$ increases from $0.2 \mathrm{~m} / \mathrm{s}$ to $0.42 \mathrm{~m} / \mathrm{s}$. In all the cases considered, a single-mode vibration and a distinct standing wave response indicated by definite nodes and antinodes are 
observed in both the IL and CF directions. These phenomena agree with the general conclusion of Constantindes and Oakley (2009) that a single mode will yield a pure standing wave response.

\subsection{Root mean square amplitudes}

The rms amplitudes in the IL and CF directions $\left(A_{x r m s} / D\right.$ and $\left.A_{y r m s} / D\right)$ are compared with the experimental data as shown in Fig. 8. The experimental data are plotted in dots for easy identification. In the case \# 1103, the maximum IL rms amplitude is found near the top end of the riser with its value $A_{x r m s}^{\max } / D \approx 0.13$ being overpredicted in the present simulation. The numerical results in the $\mathrm{CF}$ direction are in good agreement with the experimental data. The maximum $\mathrm{CF}$ rms amplitude is $A_{y r m s}^{\max } / D \approx 0.4$. When the uniform flow velocity increases to $V=0.42 \mathrm{~m} / \mathrm{s}$, the maximum IL rms amplitude increases slightly to $A_{x r m s}^{\max } / D \approx 0.14$ and appears near the bottom end of the riser at $z / L=0.22$. In contrast, the maximum $\mathrm{CF}$ rms amplitude increases dramatically to $A_{y r m s}^{\max } / D \approx 0.81$ which is similar to the CF VIV amplitudes reported by Vandiver (1993) and Huera-Huarte and Bearman (2011).

Fig. 8 (c) and Fig. 8 (d) show the comparison of the rms amplitudes between the present numerical simulation and the experiment for linearly sheared flow. Similar to the uniform flow conditions, the maximum IL and CF rms amplitudes also increase with the maximum velocity $V_{\max }$ in the two linearly sheared flow cases. However, the maximum rms amplitudes in the two linearly sheared flow cases are much smaller compared to those in the two uniform flow cases with $A_{x r m s}^{\max } / D \approx 0.03$ and $A_{y r m s}^{\max } / D \approx 0.18$ for $V_{\max }=0.2 \mathrm{~m} / \mathrm{s}$ and $A_{x r m s}^{\max } / D \approx 0.1$ and $A_{y r m s}^{\max } / D \approx 0.31$ for $V_{\max }=0.42 \mathrm{~m} / \mathrm{s}$.

\subsection{Displacement time histories and oscillation frequencies}

Fig. 9 shows the comparison of the displacement time histories at $z / L=0.22$ between the present simulation and the experiment. In general, the numerical results are comparable with the experimental data. Because the onset of the vibration occurs randomly in the numerical simulation and the experiment, there are some phase differences between the numerical and experimental time histories. 
Fig. 10 shows the comparison of the IL and CF oscillation frequencies at $z / L=0.22$. The predicted results show good agreement with the experimental data. In the present simulation, the ratio of the IL oscillation frequency to the CF oscillation frequency is around two, which conforms to the scenario of dual resonance. It is also found from the present results that the IL and CF oscillation frequencies increase with the flow velocity. In the two uniform flow cases, the IL and CF oscillation frequencies increase from $f_{\text {ox }}=3.516 \mathrm{~Hz}$ and $f_{\text {oy }}=1.953 \mathrm{~Hz}$ to $f_{o x}=6.25 \mathrm{~Hz}$ and $f_{\text {oy }}=3.125 \mathrm{~Hz}$ when $V$ increases from $0.2 \mathrm{~m} / \mathrm{s}$ to $0.42 \mathrm{~m} / \mathrm{s}$. Similarly, in the two linearly sheared flow cases, the IL and CF oscillation frequencies increase from $f_{o x}=$ $3.125 \mathrm{~Hz}$ and $f_{\text {oy }}=1.563 \mathrm{~Hz}$ to $f_{o x}=5.469 \mathrm{~Hz}$ and $f_{o y}=2.734 \mathrm{~Hz}$ when $V_{\max }$ increases from $0.2 \mathrm{~m} / \mathrm{s}$ to $0.42 \mathrm{~m} / \mathrm{s}$. The predicted IL and CF oscillation frequencies in the two linearly sheared flow cases are slightly lower than those in the two uniform flow cases. A quantitative difference in the IL oscillation frequency between the present results and the experimental data is observed in the case \# 1201 (Fig. 10 (c)). The IL oscillation frequency in the present simulation is $f_{o x}=3.125 \mathrm{~Hz}$ while it is $f_{o x}=4.73 \mathrm{~Hz}$ in the experiment. The difference in the IL oscillation frequency leads to the discrepancy in the IL dominant mode as mentioned in Subsection 4.1. The possible reason for the difference in the IL oscillation frequency might be due to the fact that the displacements in the present simulation are obtained directly whereas the displacements in the experiment are derived from the accelerations, which may increase the uncertainties during the integration process.

The predicted displacements and oscillation frequencies along the riser are further examined for all the cases. It is found that the motion at a single frequency and in a single mode shape is typical for the CF response of the riser, and a third harmonic frequency component at three times the first harmonic frequency is observed in the CF response at some locations along the riser. Fig. 11 (a) and Fig. 11 (b) show the predicted displacements and oscillation frequencies along the riser in the case \# 1103. The IL and CF responses have consistent dominant frequencies around $3.516 \mathrm{~Hz}$ and $1.953 \mathrm{~Hz}$ along the riser which correspond to the structure's second mode and first mode, respectively. The 2:1 IL to CF oscillation frequency ratio indicates the occurrence of dual resonance. In addition, a third harmonic frequency component is observed in the CF response at some locations along the riser. Fig. 11 (c) and Fig. 11 (d) are the predicted displacements and oscillation frequencies along the riser in the case \# 1201. The CF response is also consistent along the entire riser at a frequency of $f_{\text {oy }}=$ $1.563 \mathrm{~Hz}$ and again a third harmonic frequency component is found in the CF response at 
some locations along the riser. It is notable that in this case the IL response at some locations has appreciable contributions at both twice the CF response frequency and the CF response frequency itself. Similar IL response has been reported by Tognarelli et al. (2004).

\subsection{Orbital trajectories}

The predicted orbital trajectories at eight different positions along the riser span $(z / L=0.11$, $0.22,0.33,0.44,0.55,0.66,0.77$ and 0.88 ) are compared with the experimental data in Fig. 12. The selected positions coincide with the locations of the accelerometers in the experiment. As is seen from Fig. 12, most of the orbital trajectories are of a figure-eight shape indicating the occurrence of dual resonance where the IL and CF vibration frequencies have a ratio of two (Dahl et al., 2010). Another interesting phenomenon is that most of the orbital trajectories are counterclockwise (CC), i.e., the cylinder motion is counterclockwise at the top of the figure-eight motion. The exceptional clockwise $(\mathrm{C})$ trajectories are marked with letter "C" in Fig. 12. According to Bourguet et al. (2011b), the CC direction is the predominant orbit orientation in the lock-in region. The upstream motion of the cylinder in the CC trajectory leads to a closer proximity of the cylinder and the recently shed vortices and energy is transferred from the fluid to the body under a resonance condition (Dahl et al., 2007). On the other hand, clockwise orbits are associated with damping fluid forces (Bourguet et al., 2011b). In the cases considered, most of the riser span is in the lock-in region regardless of the incoming flow conditions. According to the orbit orientations, it can be seen that the nonlock-in regions in the two linearly sheared flow cases are larger than those in the two uniform flow cases.

\subsection{Vortex shedding modes}

Fig. 13 shows the vortex shedding at five different planes along the riser, i.e., $z / L=0.11,0.3$, $0.49,0.68$ and 0.88 under different current profiles. It can be seen from the vorticity contour plots that the majority of the vortex shedding shows a clear $2 \mathrm{~S}$ pattern (two single vortices per cycle), whereas a $2 \mathrm{P}$ mode (two vortex pairs formed in each cycle of the body motion) is

observed in the case $\# 1105$ at $z / L=0.3$ near the position $z / L=0.22$ where the maximum vibration amplitude appears. The present observation agrees with the conclusions of Meneghini et al. (2004), Yamamoto et al. (2004) and Sun et al. (2012) for VIV of flexible 
risers that a $2 \mathrm{~S}$ mode is found in regions of small amplitudes and a $2 \mathrm{P}$ mode is observed in regions of larger amplitudes.

\subsection{Fatigue damage indices}

One of the objectives to study flexible riser VIV is to assess the fatigue damage caused by the vibration, thus the fatigue damage index as defined by Tognarelli et al. (2004) is evaluated in this subsection with available numerical modelling results. The damage index is defined as $D I=f_{z, \varepsilon} \varepsilon^{3}$ where $f_{z, \varepsilon}$ is the zero-crossing frequency of the bending strain and $\varepsilon$ is the rms strain. In the present simulation, the strain data in the IL and CF directions are obtained from ANSYS software. The calculated fatigue damage indices are compared to the experimental data in Fig. 14. The comparison shows that the present numerical results are generally in good agreement with the experimental data. As is shown in Fig. 14, the IL and CF fatigue damage indices increase with the flow velocity in all the cases considered. According to Subsection 4.3, the response frequencies in the case \# 1105 are slightly higher than those in the case \# 1205, however, the fatigue damage indices in the case \# 1105 are considerably higher than those in the case \# 1205 in the lower part of the riser. That is because the largeamplitude vibration associated by the $2 \mathrm{P}$ vortex shedding mode produces significantly larger strains in the IL and CF directions. According to Tognarelli et al. (2004), the fatigue damage index is determined by the mode number, the response frequency and the response amplitude. As there are discrepancies in the IL dominant mode number and response frequency in the case \# 1201 between the present numerical results and the experimental data, a discrepancy is also observed in the IL fatigue damage index. It is noteworthy that in all the four cases considered in the present simulation, the IL fatigue damage is in fact higher than CF fatigue damage in the two low flow velocity cases, i.e., \# 1103 and \# 1201. It is only when the flow velocity or maximum flow velocity increases to $0.42 \mathrm{~m} / \mathrm{s}$ that the $\mathrm{CF}$ fatigue damage reaches the same order of magnitude as the IL fatigue damage. This finding agrees with the conclusion of Tognarelli et al. (2004). Although the present numerical simulation does not cover as many velocities as the experiment did, the available results have already suggested that, from a design point of view, the IL fatigue damage is not negligible especially for low flow velocity or low mode number applications such as pipeline spans or some drilling and production riser. 


\section{Conclusions}

VIV of a vertical riser in the uniform and linearly sheared currents is numerically studied using a fully 3D FSI simulation methodology. The results of a total of four cases are presented for two uniform flow profiles with $V=0.2 \mathrm{~m} / \mathrm{s}$ and $0.42 \mathrm{~m} / \mathrm{s}$ and two linearly sheared flow profiles with $V_{\max }=0.2 \mathrm{~m} / \mathrm{s}$ and $0.42 \mathrm{~m} / \mathrm{s}$ at the bottom end and $V_{\min } / V_{\max }=0.14$. The predicted numerical results are in good agreement with the ExxonMobil vertical riser model test results. The overall comparison indicates that the present numerical method is reliable and capable of predicting reasonably accurate VIV responses of long risers subject to uniform currents and linearly sheared currents. In addition, with our numerical modelling, flow visualisation results which are hard to obtain in the experimental tests are provided. The main findings of the present paper can be summarised as follows.

The dynamic response of the riser is studied by examining the dominant modes, rms amplitudes, displacement time histories, dominant frequencies and orbital trajectories. It is found that the dominant mode numbers in the IL and CF directions increase with the flow velocity. The variation of the dominant modes in the two linearly sheared flow cases is the same as that in the two uniform flow cases. A single-mode vibration and a distinct standing wave response are observed in both the IL and CF directions. In terms of the rms amplitudes, the maximum IL and CF rms amplitudes are found to increase with the flow velocity under both flow conditions. However, the maximum rms amplitudes in the two linearly sheared flow cases are much smaller than those in the two uniform flow cases. In general, the IL response has a dominant frequency twice the $\mathrm{CF}$ response frequency. Both the $\mathrm{IL}$ and $\mathrm{CF}$ oscillation frequencies increase with the flow velocity. In the meanwhile, the IL and CF oscillation frequencies in the two linearly sheared flow cases are slightly lower than those in the two uniform flow cases. The motion at a single frequency and in a single mode shape is typical for the CF response of the riser. A third harmonic CF frequency component is found at some locations along the riser. In certain cases, the IL response at some locations has appreciable contributions at both twice the CF response frequency and the CF response frequency itself. As for the orbital trajectories, most of the orbital trajectories are of a figureeight shape indicating the occurrence of dual resonance and most of the riser span is in the lock-in region characterised by counterclockwise orbits. Judging from the orbit orientations, the two linearly sheared flow cases have larger non-lock-in regions than the two uniform flow cases. 
As vortex shedding is an important aspect of flexible riser VIV analysis, the vortex shedding modes at different slices along the riser span are studied. Two different vortex shedding modes are observed in the present simulation, i.e., $2 \mathrm{~S}$ and $2 \mathrm{P}$ modes. A $2 \mathrm{~S}$ mode is widely observed in all the cases considered, whereas a $2 \mathrm{P}$ mode is found to be associated with the maximum amplitude.

The fatigue damage due to VIV is analysed in the consideration of its significance in practical applications. It is found that the IL and CF fatigue damage indices increase with the flow velocity as a result of the increased mode number, the increased response frequency and the increased response amplitude. The larger-amplitude vibration associated by the $2 \mathrm{P}$ vortex shedding mode in the lower part of the riser in the case \# 1105 produces significantly larger strains in the IL and CF directions leading to considerably higher fatigue damage indices. The IL fatigue damage is higher than the CF fatigue damage at low flow velocities in the cases \# 1103 and \# 1201. The CF fatigue damage reaches the same order of magnitude as the IL fatigue damage in the cases \# 1105 and \# 1205. The results emphasise the importance of the IL fatigue damage especially for low flow velocity or low mode number applications.

As this paper mainly focusses on a low flow velocity range and only the uniform flow and linearly sheared flow cases are considered. Future research on riser VIV in a higher flow velocity range under more complex flow conditions is worthwhile.

Overall, the present numerical method is able to reasonably predict VIV response of a vertical riser under uniform and linearly sheared flow conditions and can be used as an altenative to the existing prediction models for deepwater riser VIV prediction.

\section{Acknowledgements}

Results were obtained using the EPSRC funded ARCHIE-WeSt High Performance Computer (www.archie-west.ac.uk). EPSRC grant no. EP/K000586/1.

\section{References}

ANSYS Inc., 2013. ANSYS CFX-Solver Theory Guide, Canonsburg, USA. 
Bearman, P.W., 1984. Vortex shedding from oscillating bluff body. Annual Review of Fluid Mechanics 16, 195 - 222.

Bearman, P.W., 2011. Circular cylinder wakes and vortex-induced vibrations. Journal of Fluids and Structures 27, 648 - 658.

Blevins, R.D., 1977. Flow-Induced Vibration. Van Nostrand Reinhold Company, New York, USA.

Bourguet, R., Karniadakis, G.E., Triantafyllou, M.S., 2011a. Lock-in of the vortex-induced vibrations of a long tensioned beam in shear flow. Journal of Fluids and Structures 27, $838-847$.

Bourguet, R., Modarres-Sadeghi, Karniadakis, G.E., Triantafyllou, M.S., 2011b. Wake-body resonance of long flexible structures is dominated by counterclockwise orbits. Physical Review Letters 107 (134502), 1 - 4.

Bourguet, R., Karniadakis, G.E., Triantafyllou, M.S., 2013. Phasing mechanisms between the in-line and cross-flow vortex-induced vibrations of a long tensioned beam in shear flow. Computers \& Structures 122, 155 - 163.

Chaplin, J.R., Bearman, P.W., Huera Huarte, F.J., Pattenden, R.J., 2005. Laboratory measurement of vortex-induced vibrations of a vertical tension riser in a stepped current. Journal of Fluids and Structures 21, 3 - 24.

Chung, J., Hulbert, G.M., 1993. A time integration algorithm for structural dyanmics with improved numerical dissipation: the generalised $-\alpha$ method. Journal of Applied Mechanics 60, 371 - 375.

Constantindes, Y., Oakley, O.H., 2009. Numerical simulation of cylinder VIV focusing on high harmonics, OMAE2009-800002, In: Proceedings of the ASME 28th International Conference on Ocean, Offshore and Arctic Engineering, Honolulu, USA.

Constantinides, Y., Oakley, O.H., 2008. Numerical prediction of VIV and comparison with field experiments, OMAE2008-57215, In: Proceedings of the ASME 27th International Conference on Ocean, Offshore and Arctic Engineering, Estoril, Portugal.

Dahl, J.M., Hover, F.S., Triantafyllou, M.S., 2007. Resonant vibrations of bluff bodies cause multivortex shedding and high frequency forces. Physical Review Letters 99 (144503), 1 $-4$.

Dahl, J.M., Hover, F.S., Triantafyllou, M.S., Oakley, O.H., 2010. Dual resonance in vortexinduced vibrations at subcritical and supercritical Reynolds numbers. Journal of Fluid Mechanics 643, 395 - 424. 
Evangelinos, C., Karniadakis, G.E., 1999. Dynamics and flow structures in the turbulent of rigid and flexible cylinders subject to vortex-induced vibrations. Journal of Fluid Mechanics 400, 91 - 124.

Gabbai, R.D., Benaroya, H., 2005. An overview of modelling and experiments of vortexinduced vibrations of circualr cylinders. Journal of Sound and Vibration 282, 575 - 646.

Gao, Y., Fu, S., Ren, T., Xiong, Y., Song, L., 2015. VIV response of a long flexible riser fitted with strakes in uniform and linearly sheared currents. Applied Ocean Research 52, $102-114$

Gu, J., Vitola, M., Coelho, J., W., P., Duan, M., Levi, C., 2013. An experimental investigation by towing tank on VIV of a long flexible cylinder for deep water riser application. Journal of Marine Science and Technology 18, 358 - 369.

Holmes, S., Oakley, O.H., Constantinides, Y., 2006. Simulation of riser VIV using fully three dimensional CFD simulations, OMAE2006-92124, In: Proceedings of the ASME 25th International Conference on Ocean, Offshore and Arctic Engineering, Hamburg, Germany.

Huang, K., Chen, H.C., Chen, C.R., 2009. Vertical riser VIV simulation in uniform currents. Journal of Offshore Mechanics and Arctic Engineering 132, 1 - 10.

Huang, K., Chen, H.C., Chen, C.R., 2011a. Numerical scheme for riser motion calculation during 3D VIV simulation. Journal of Fluids and Structures 27, 947 - 961.

Huang, S., Khorasanchi, M., Herfjord, K., 2011b. Drag amplification of long flexible riser models undergoing multi-mode VIV in unifrom currents. Journal of Fluids and Structures $27,342-353$.

Huera-Huarte, F.J., Bearman, P.W., 2011. Vortex and wake-induced vibrations of a tandem arrangement of two flexible circular cylinders with near wake interference. Journal of Fluids and Structures 27, 193 - 211.

Jhingran, V., Vandiver, J.K., 2007. Incorporating the higher harmonics in VIV fatigue predictions, OMAE2007-29352, In: Proceedings of the ASME 26th International Conference on Offshore Mechanics and Arctic Engineering, San Diego, USA.

Lehn, E., 2003. VIV Suppression Tests on High L/D Flexible Cylinders. Norwegian Marine Technology Research Institute, Trondheim, Norway.

Lie, H., Kaasen, K.E., 2006. Modal analysis of measurements from a large-scale VIV model test of a riser in linearly sheared flow. Journal of Fluids and Structures 22, 557 - 575. 
Meneghini, J.R., Saltara, F., R.A., F., Yamamoto, C.T., Casaprima, E., Ferrari, J.A., 2004. Numerical simulation of VIV on long flexible cylinders immersed in complex flow fields. European Journal of Mechanics-B/Fluids 23, 51 - 63.

Menter, F., Sharkey, P., Yakubov, S., Kuntz, M., 2006. Overview of fluid-structure coupling in ANSYS-CFX, OMAE2006-92145, In: Proceedings of the ASME 25th International Conference on Ocean, Offshore and Arctic Engineering, Hamburg, Germany.

Newman, D.J., Karniadakis, G.E., 1997. A direct numerical simulation study of flow past a freely vibrating cable. Journal of Fluid Mechanics 344, 95 - 136.

Nicoud, F., Ducros, F., 1999. Subgrid-scale stress modelling based on the square of the velocity gradient tensor. Flow, Turbulence and Combustion 62, 183 - 200.

Sarpkaya, T., 1979. Vortex-induced oscillations. Journal of Applied Mechanics 46, 241 - 258.

Sun, L., Zong, Z., Dong, J., Dong, G.H., Liu, C.F., 2012. Stripwise discrete vortex method for VIV analysis of flexible risers. Journal of Fluids and Structures 35, 21 - 49.

Tognarelli, M.A., Slocum, S.T., Frank, W.R., Campbell, R.B., 2004. VIV response of a long flexible cylinder in uniform and linearly sheared currents, OTC 16338, In: Proceedings of the 2004 Offshore Technology Conference, Houston, USA.

Tognarelli, M.A., Taggart, S., Campbell, M., 2008. Actual VIV fatigue response of full scale drilling risers: with and without suppresion devices, OMAE2008-57046, In: Proceedings of the ASME 27th International Conference on Ocean, Offshore and Arctic Engineering, Estoril, Portugal.

Trim, A.D., Braaten, H., Lie, H., Tognarelli, M.A., 2005. Experimental investigation of vortex-induced vibration of long marine risers. Journal of Fluids and Structures 21335 361.

Vandiver, J.K., 1993. Dimensionless parameters important to the prediction of vortexinduced vibration of long, flexible cylinders in ocean currents. Journal of Fluids and Structures 7, 423 - 455.

Vandiver, J.K., Swithenbank, S., Jaiswal, V., Marcollo, H., 2006. The effectiveness of helical strakes in the suppression of high-mode-number VIV, OTC 18276, In: Proceedings of the 2006 Offshore Technology Conference, Houston, USA.

Vandiver, J.K., Jaiswal, V., Jhingran, V., 2009. Insights on vortex-induced, travelling waves on long risers. Journal of Fluids and Structures 25, 641 - 653.

Weaver, W., Timoshenko, S.P., Young, D.H., 1974. Vibration Problems in Engineering. John Wiley \& Sons, New York, USA. 
Willden, R.H.J., Graham, J.M.R., 2001. Numerical prediction of VIV on long flexible circular cylinders. Journal of Fluids and Structures 15, 659 - 669.

Williamson, C.H.K., Govardhan, R., 2004. Vortex-induced vibrations. Annual Review of Fluid Mechanics 36, 413 - 455.

Wu, X., Ge, F., Hong, Y., 2012. A review of recent studies on vortex-induced vibrations of long slender cylinders. Journal of Fluids and Structures 28, 292 - 308.

Xue, H., Wang, K., Tang, W., 2015. A practical approach to predicting cross-flow and in-line VIV response for deepwater risers. Applied Ocean Research 52, 92 - 101.

Yamamoto, C.T., Meneghini, J.R., Saltara, F., Fregonesi, R.A., Ferrari, J.A., 2004. Numerical simulations of vortex-induced vibration on flexible cylinders. Journal of Fluids and Structures 19, 467 - 489.

Zhao, M., Cheng, L., 2011 Numerical simulation of two-degree-of-freedom vortex-induced vibration of a circular cylinder close to a plane boundary Journal of Fluids and Structures $27,1097-1110$.

Zhao, M., Cheng, L., An, H., Lu, L., 2014. Three-dimensional numerical simulation of vortex-induced vibration of an elastically mounted circular cylinder in steady current. Journal of Fluids and Structures 50, 292 - 311. 


\section{List of Tables}

Table 1 Properties of the vertical riser model.

Table 2 Incoming flow velocity parameters of different cases.

Table 3 Eigenfrequencies for the vertical riser model.

Table 4 Mesh dependence test results. 


\section{List of Figures}

Fig. 1 (a) Sketch of physical configurations and (b) Uniform and linearly sheared incoming flow velocity profiles.

Fig. 2 Flow chart of two-way explicit FSI solution procedures (for one time step).

Fig. 3 (a) Computational domain, (b) computational mesh in the $x y$-plane, (c) mesh around the cylinder, (d) initial mesh and (e) mesh with riser deflection.

Fig. 4 FEA mesh: (a) initial mesh and (b) mesh with riser deflection.

Fig. 5 Comparison of the rms amplitudes between different mesh systems with published data:

(a) IL rms amplitudes and (b) CF rms amplitudes.

Fig. 6 Comparison of the riser response envelopes: (a) \# 1103, (b) \# 1105, (c) \# 1201 and (d) \# 1205 .

Fig. 7 The IL and CF motion evolution responses along the riser: (a) \# 1103, (b) \# 1105, (c) \# 1201 and (d) \# 1205.

Fig. 8 Comparison of the rms amplitudes: (a) \# 1103, (b) \# 1105, (c) \# 1201 and (d) \# 1205.

Fig. 9 Comparison of the displacement time histories at $z / L=0.22$ : (a) \# 1103, (b) \# 1105, (c) \# 1201 and (d) \# 1205.

Fig. 10 Comparison of the oscillation frequencies at $z / L=0.22$ : (a) \# 1103, (b) \# 1105, (c) \# 1201 and (d) \# 1205.

Fig. 11 Displacements and oscillation frequencies along the riser: (a) displacement time histories (\# 1103), (b) oscillation frequencies (\# 1103), (c) displacement time histories (\# 1201) and (d) oscillation frequencies (\#1201).

Fig. 12 Comparison of the orbital trajectories at various positions: (a) \# 1103, (b) \# 1105, (c) \# 1201 and (d) \# 1205.

Fig. 13 Vortex shedding under different current profiles: (a) \# 1103, (b) \# 1105, (c) \# 1201 and (d) \# 1205.

Fig. 14 Comparison of the fatigue damage indices: (a) \# 1103, (b) \# 1105, (c) \# 1201 and (d) \# 1205 . 
Table 1 Properties of the vertical riser model.

\begin{tabular}{lll}
\hline Properties & Values & SI units \\
\hline$L$ & 9.63 & $\mathrm{~m}$ \\
$D_{o}$ & 20 & $\mathrm{~mm}$ \\
$t_{w}$ & 0.45 & $\mathrm{~mm}$ \\
$E$ & $1.025 \times 10^{11}$ & $\mathrm{~N} / \mathrm{m}^{2}$ \\
$T$ & 817 & $\mathrm{~N}$ \\
$m^{*}$ & 2.23 & - \\
$L / D$ & 481.5 & - \\
\hline
\end{tabular}

Table 2 Incoming flow velocity parameters of different cases.

\begin{tabular}{cccc}
\hline Case \# & Flow conditions & $V_{\max }(\mathrm{m} / \mathrm{s})$ & $V_{\min } / V_{\max }$ \\
\hline 1103 & Uniform & 0.2 & 1 \\
1105 & Uniform & 0.42 & 1 \\
1201 & Linearly Sheared & 0.2 & 0.14 \\
1205 & Linearly Sheared & 0.42 & 0.14 \\
\hline
\end{tabular}


Table 3 Eigenfrequencies for the vertical riser model.

\begin{tabular}{cccccc}
\hline Mode & $f_{n, \text { string }}$ & $f_{n, \text { beam }}$ & Theoretical Value & FEA & Error \\
\hline 1 & 1.77 & 0.24 & 1.79 & 1.7904 & $0.022 \%$ \\
2 & 3.55 & 0.94 & 3.67 & 3.6725 & $0.068 \%$ \\
3 & 5.32 & 2.12 & 5.73 & 5.7309 & $0.015 \%$ \\
4 & 7.1 & 3.77 & 8.04 & 8.0373 & $0.034 \%$ \\
5 & 8.87 & 5.89 & 10.65 & 10.649 & $0.0094 \%$ \\
6 & 10.64 & 8.48 & 13.62 & 13.61 & $0.073 \%$ \\
7 & 12.42 & 11.55 & 16.96 & 16.952 & $0.047 \%$ \\
8 & 14.2 & 15.08 & 20.71 & 20.698 & $0.058 \%$ \\
\hline
\end{tabular}

Table 4 Mesh dependence test results.

\begin{tabular}{lcccccc}
\hline & $N_{\text {node }}$ & $\Delta z / D$ & $\Delta r / D$ & $N_{\mathrm{c}}$ & $A_{x r m s}^{\max } / D$ & $A_{y r m s}^{\max } / D$ \\
\hline Mesh 1 & 626040 & 0.332 & 0.001 & 180 & 0.118 & 0.781 \\
Mesh 2 & 1043400 & 0.197 & 0.001 & 180 & 0.125 & 0.805 \\
Mesh 3 & 2086800 & 0.097 & 0.001 & 180 & 0.128 & 0.823 \\
EXP & - & - & - & - & 0.14 & 0.745 \\
Huang et al. (2011a) & 1480100 & 0.197 & $? ?$ & 182 & $? ?$ & 0.833 \\
\hline
\end{tabular}




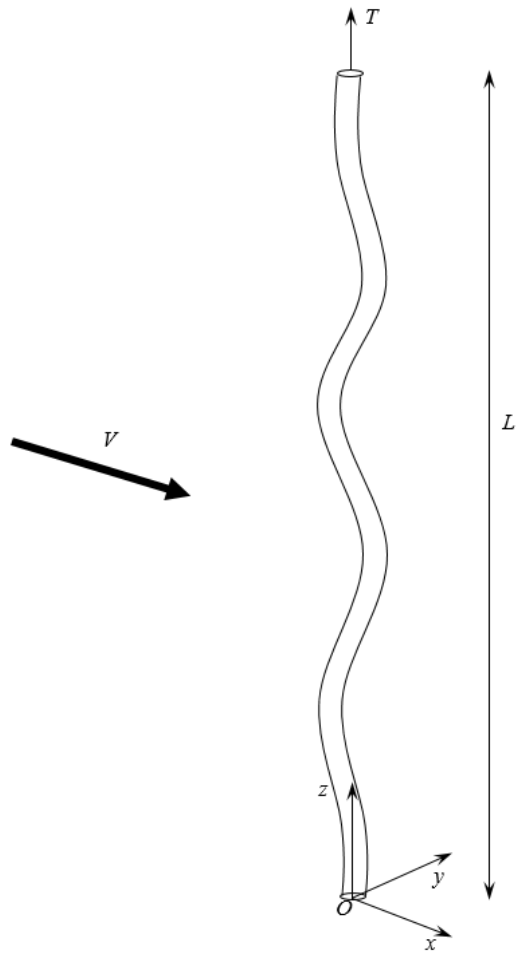

(a)

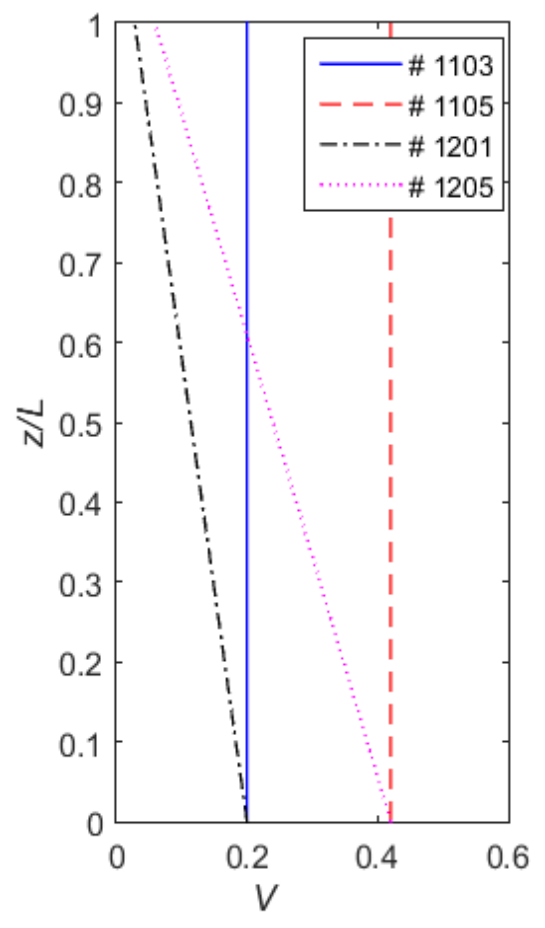

(b)

Fig. 1 (a) Sketch of physical configurations and (b) Uniform and linearly sheared incoming flow velocity profiles. 


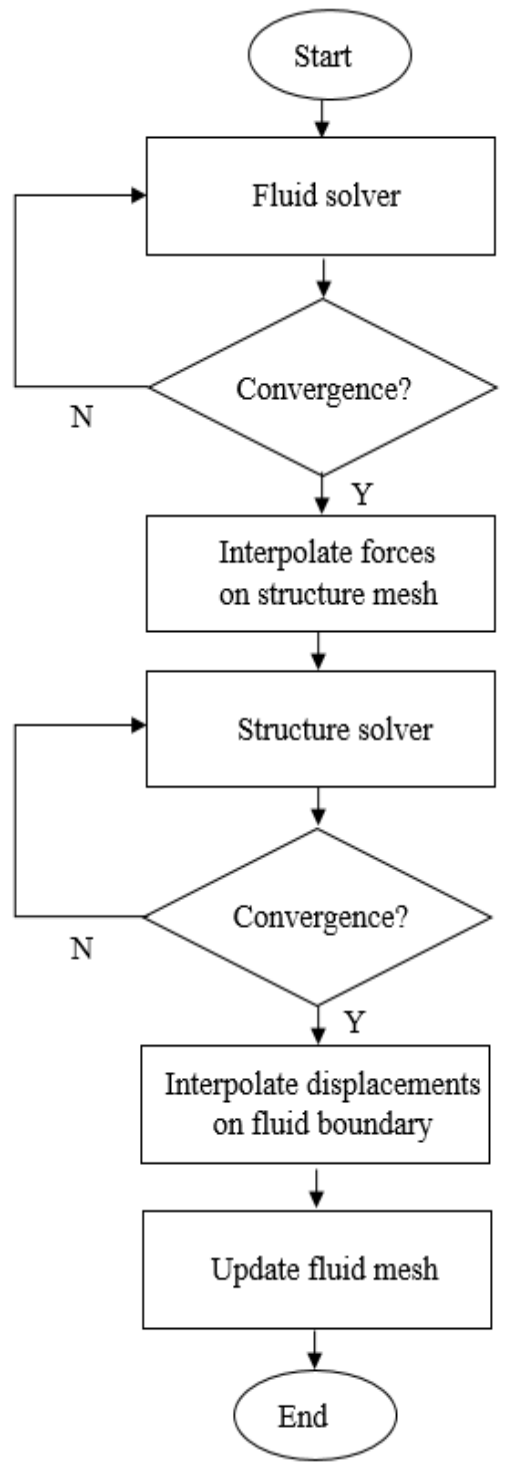

Fig. 2 Flow chart of two-way explicit FSI solution procedures (for one time step). 


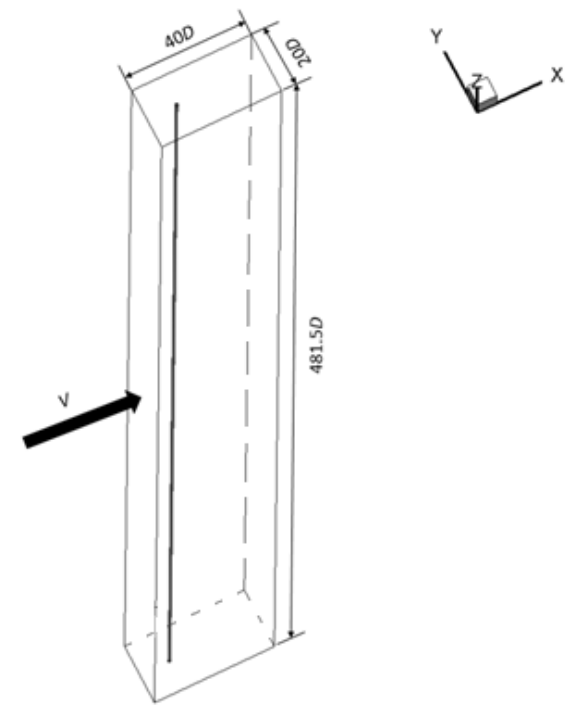

(a)

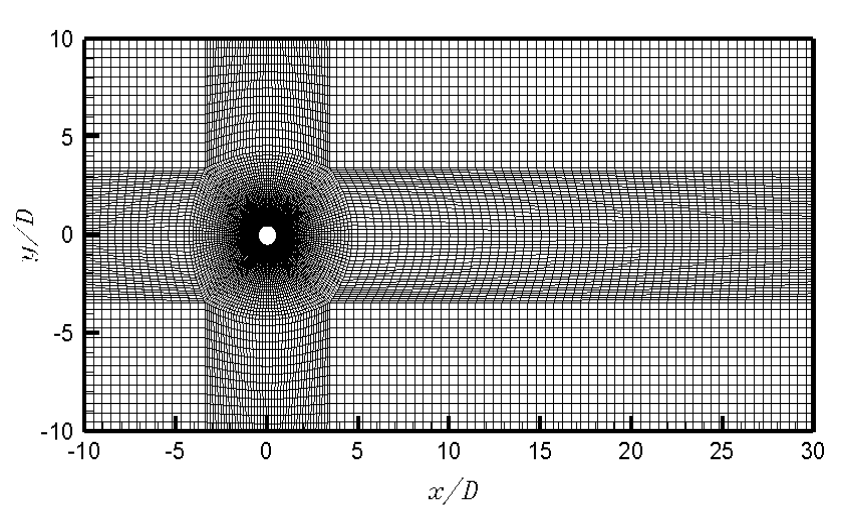

(b)

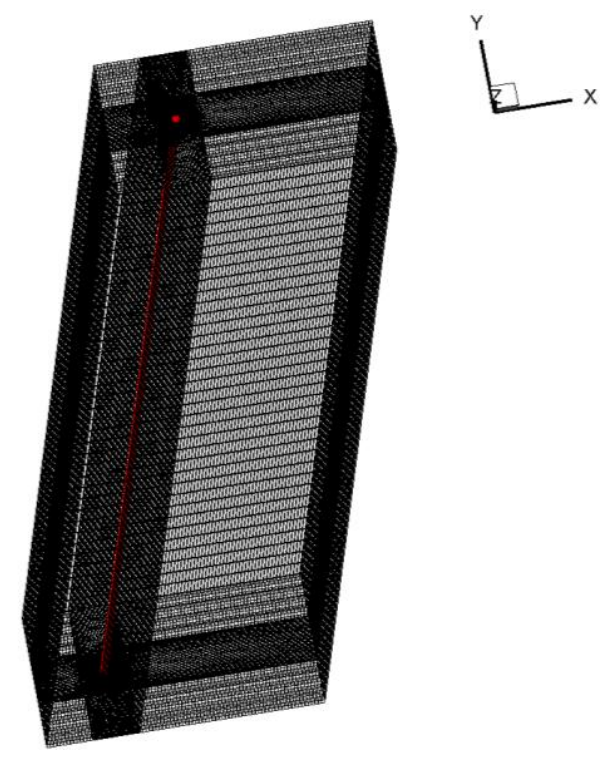

(d)

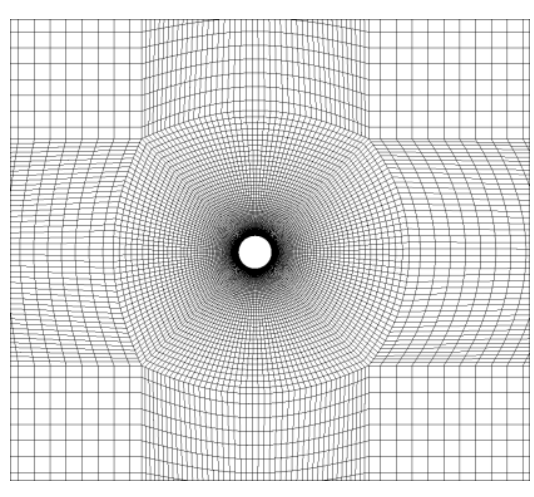

(c)

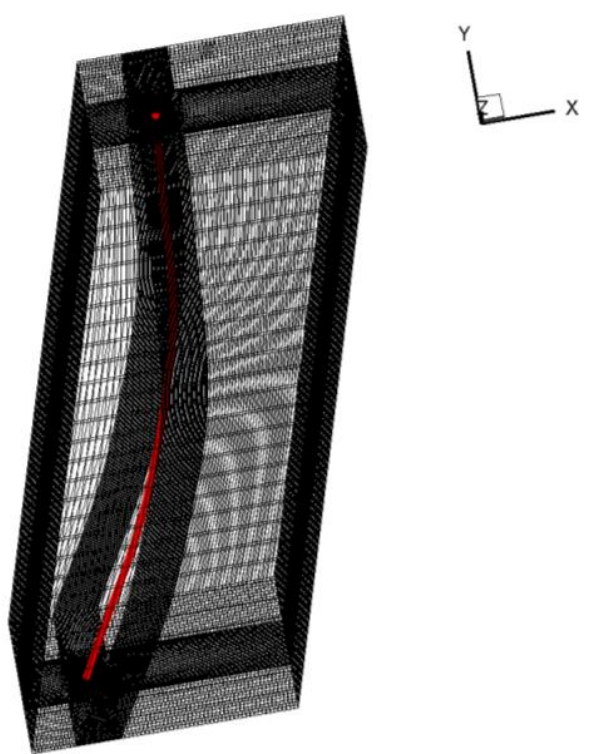

(e)

Fig. 3 (a) Computational domain, (b) computational mesh in the $x y$-plane, (c) mesh around the cylinder, (d) initial mesh and (e) mesh with riser deflection. 

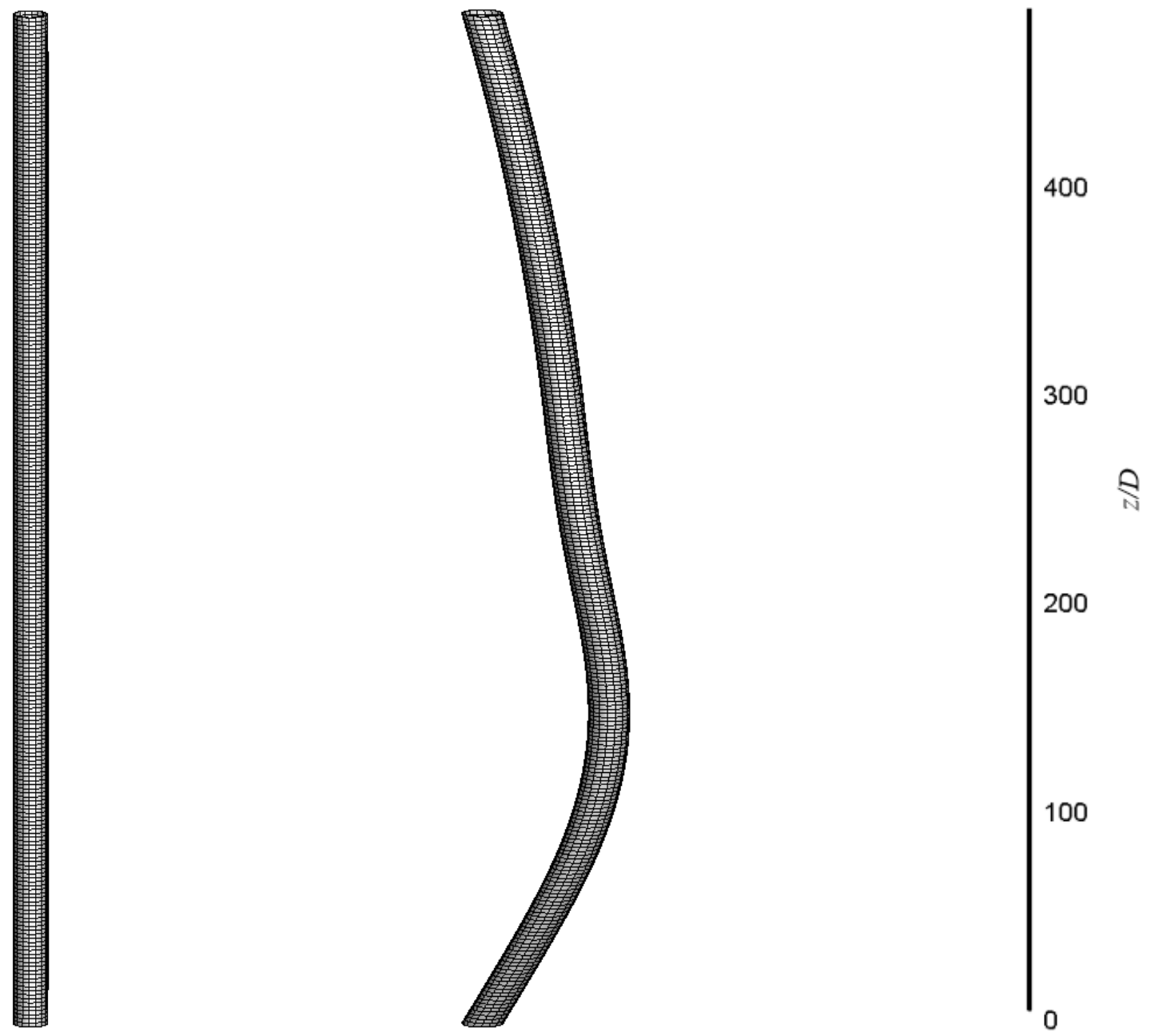

Fig. 4 FEA mesh: (a) initial mesh and (b) mesh with riser deflection. 


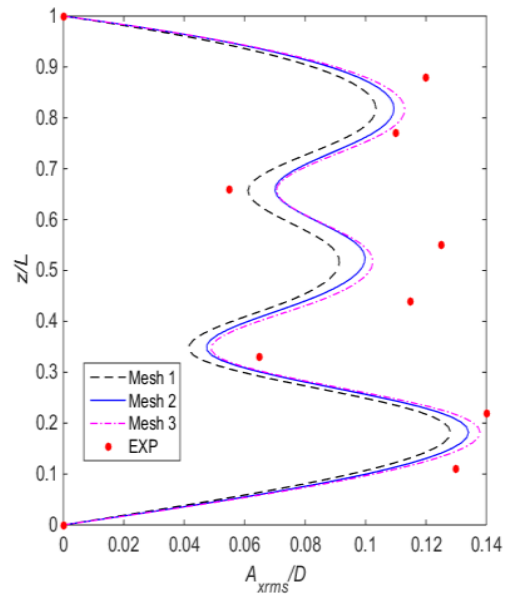

(a)

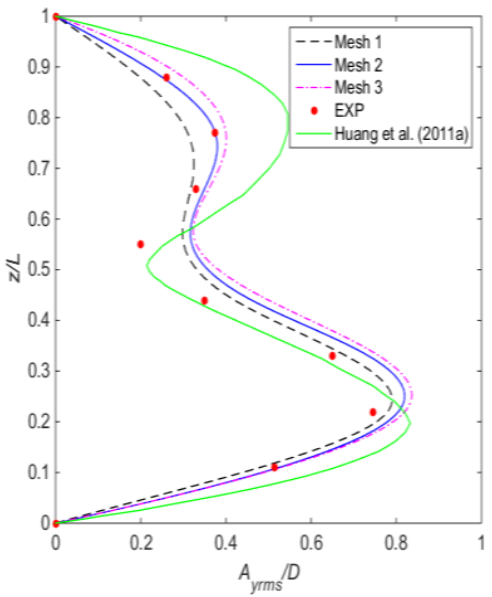

(b)

Fig. 5 Comparison of the rms amplitudes between different mesh systems with published data:

(a) IL rms amplitudes and (b) CF rms amplitudes. 

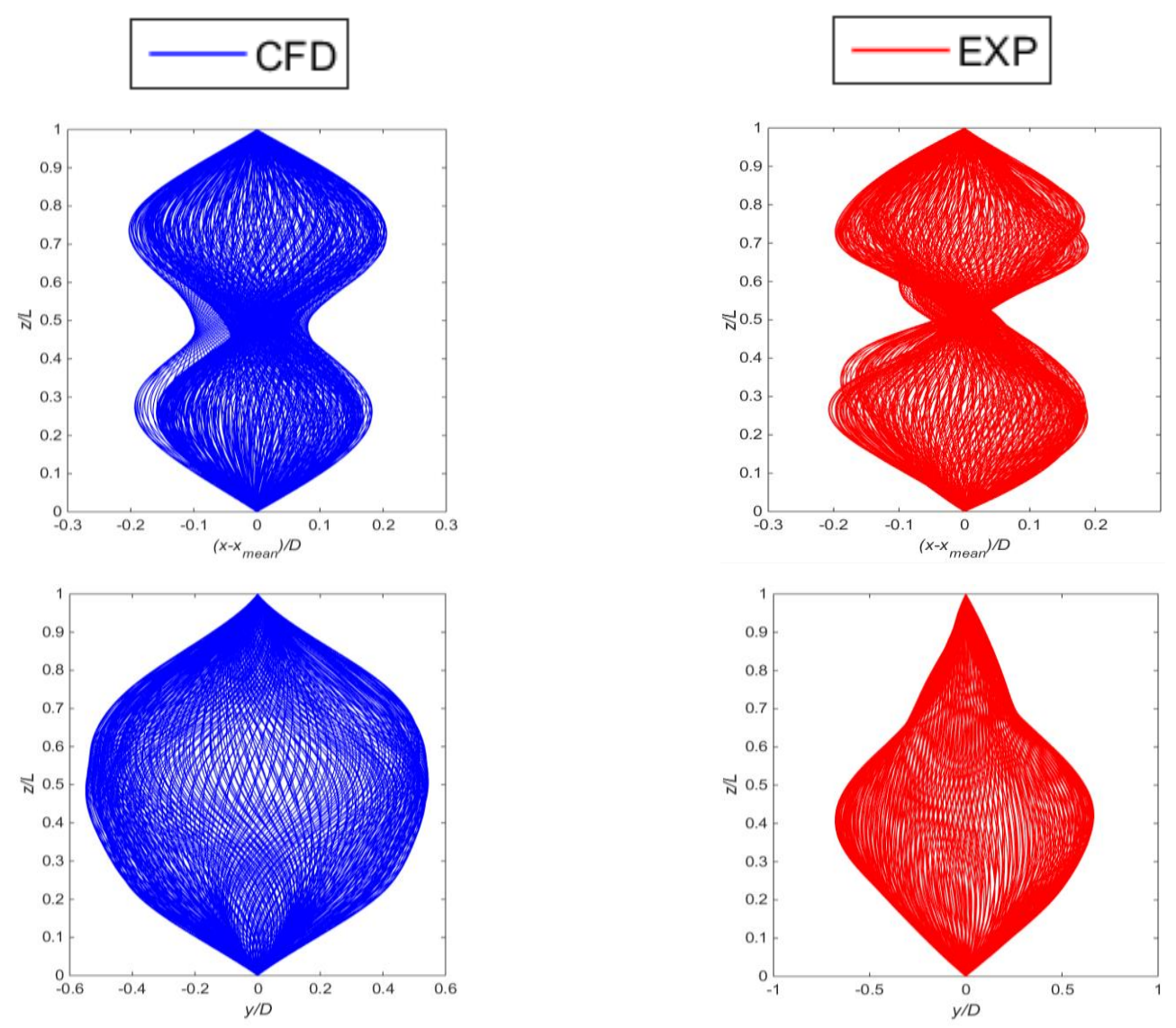

(a)
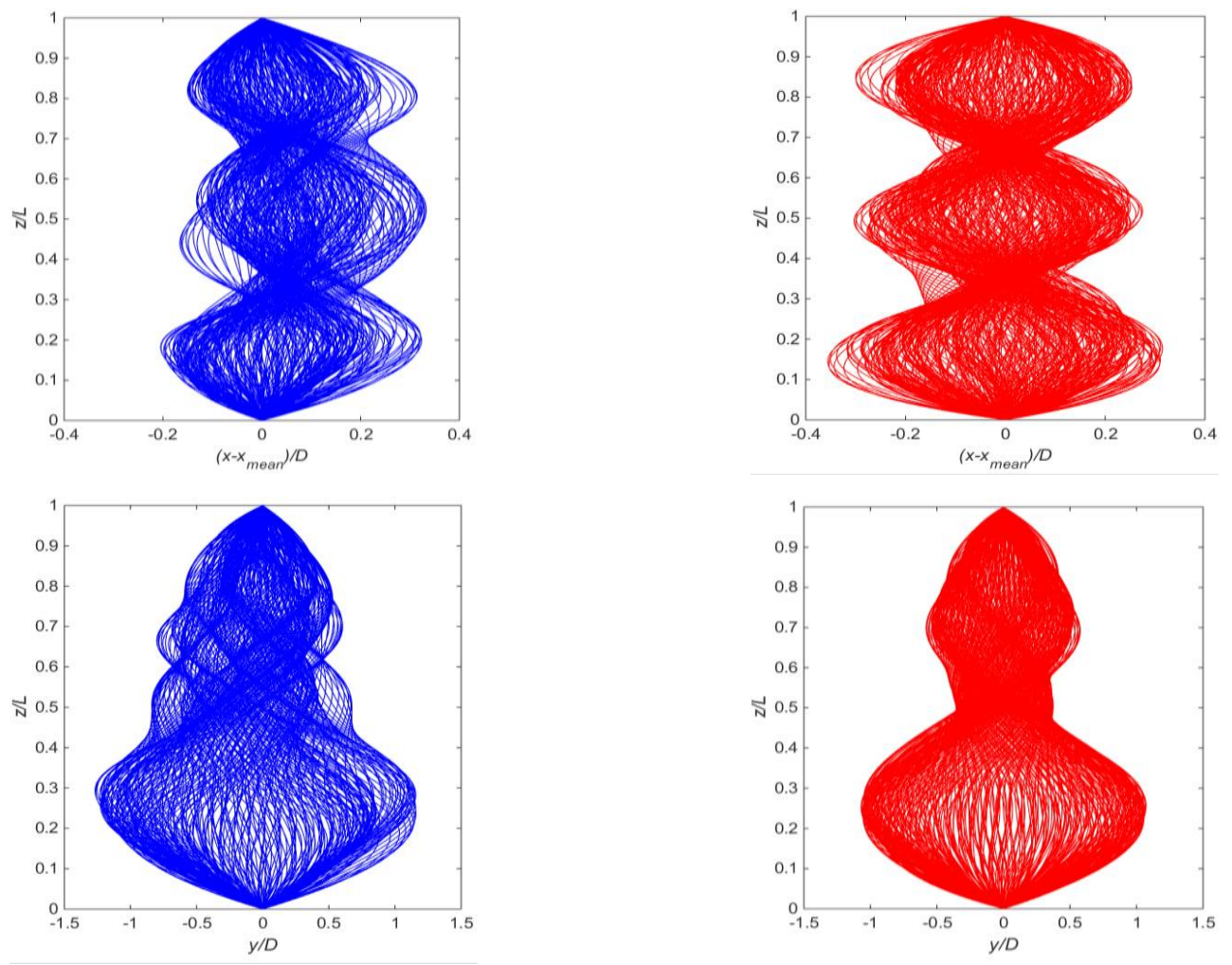

(b) 

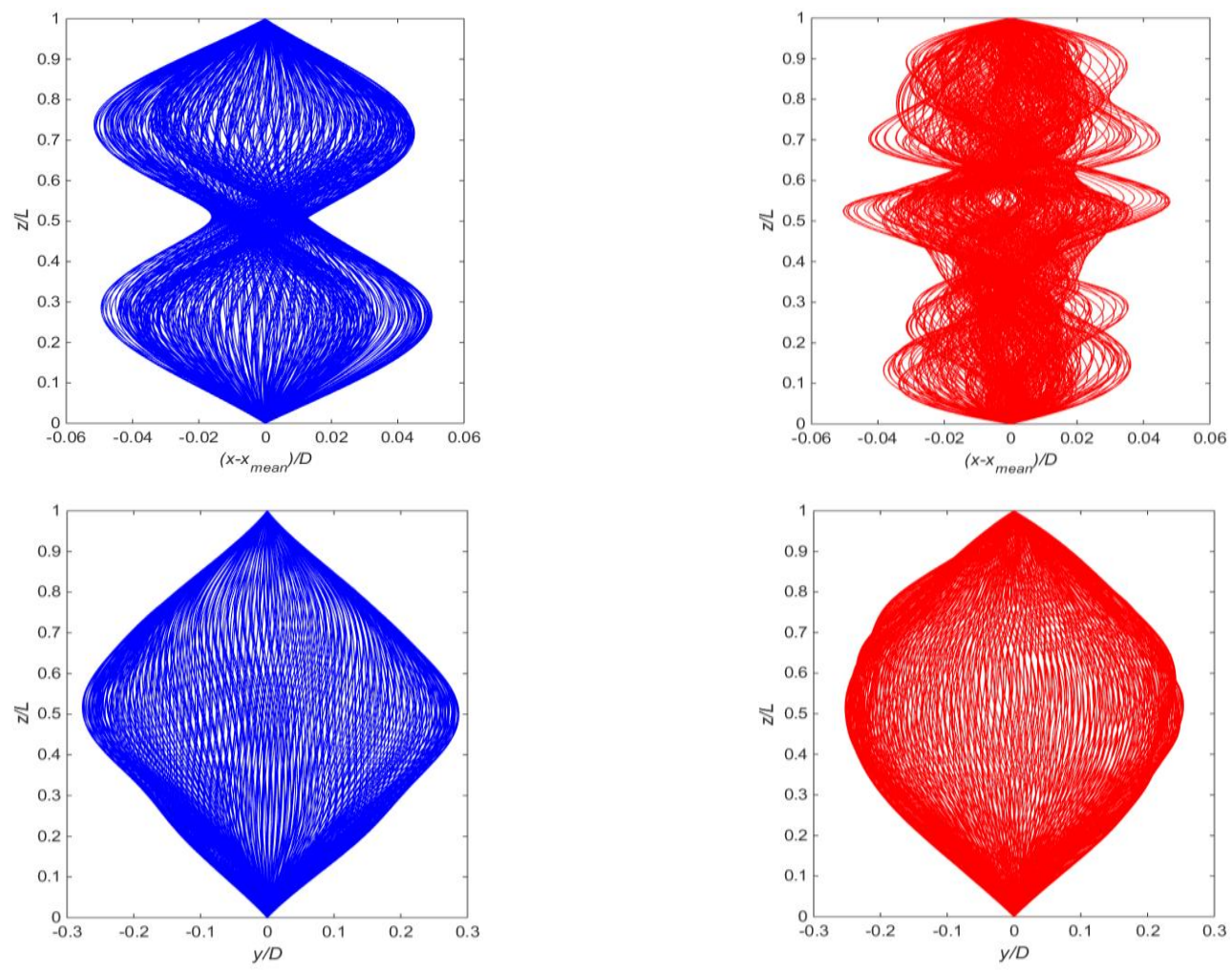

(c)
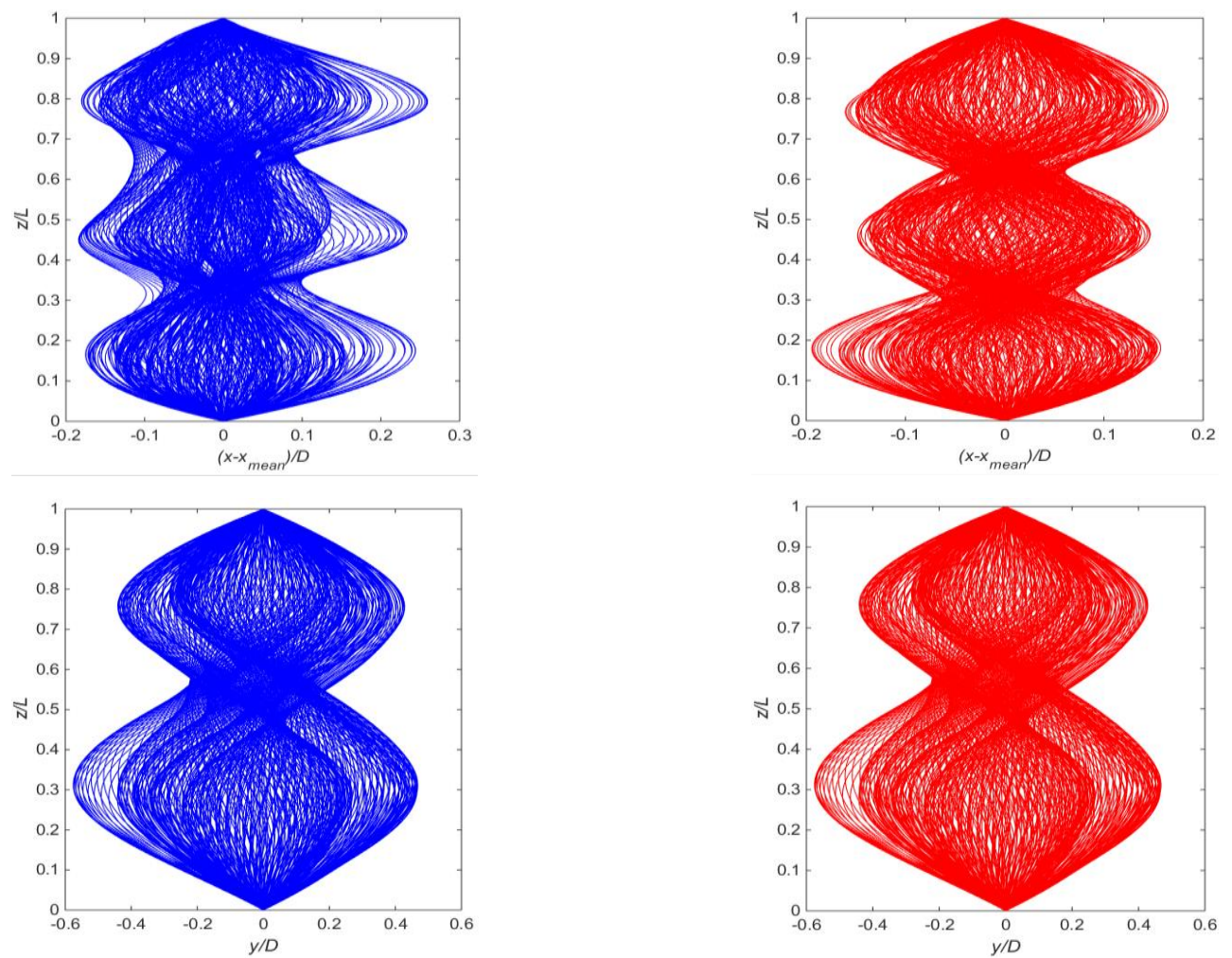

(d)

Fig. 6 Comparison of the riser response envelopes: (a) \# 1103, (b) \# 1105, (c) \# 1201 and (d) \# 1205. 

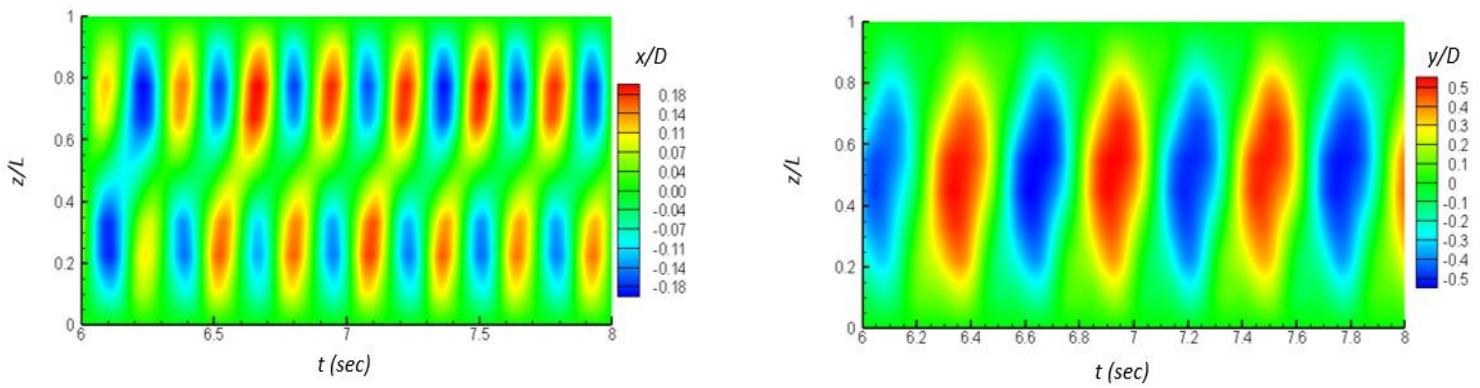

(a)
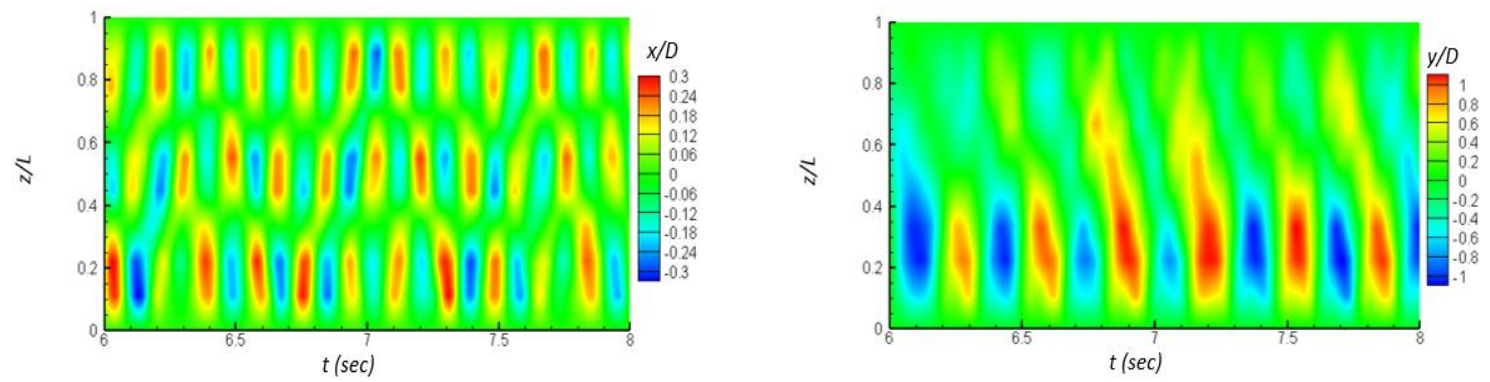

(b)
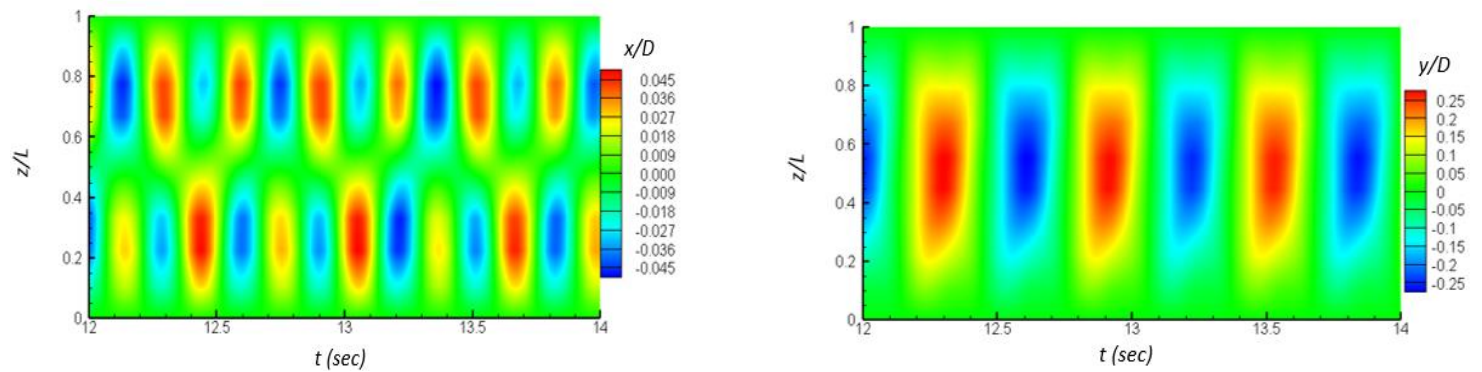

(c)
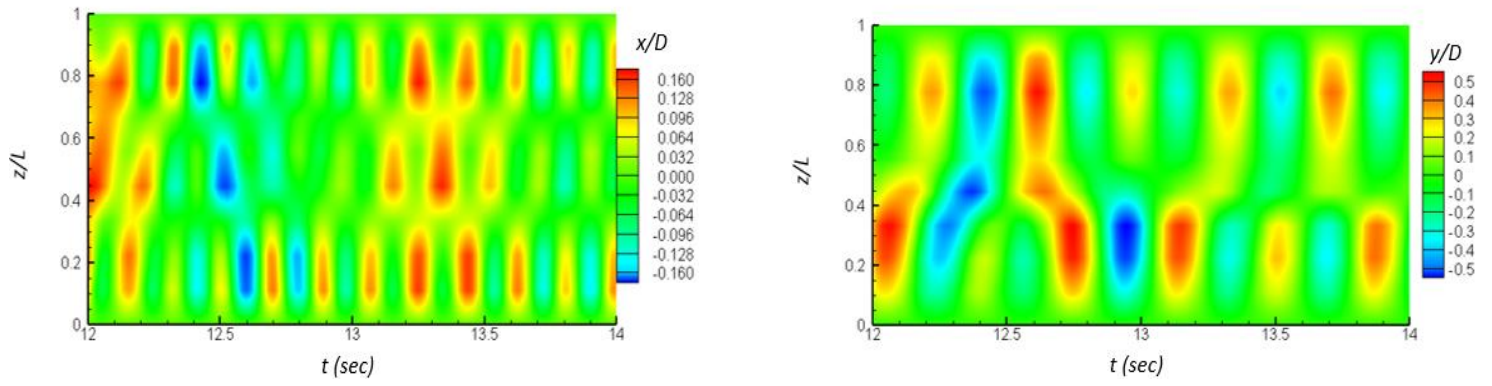

(d)

Fig. 7 The IL and CF motion evolution responses along the riser: (a) \# 1103, (b) \# 1105, (c) \# 1201 and (d) \# 1205. 

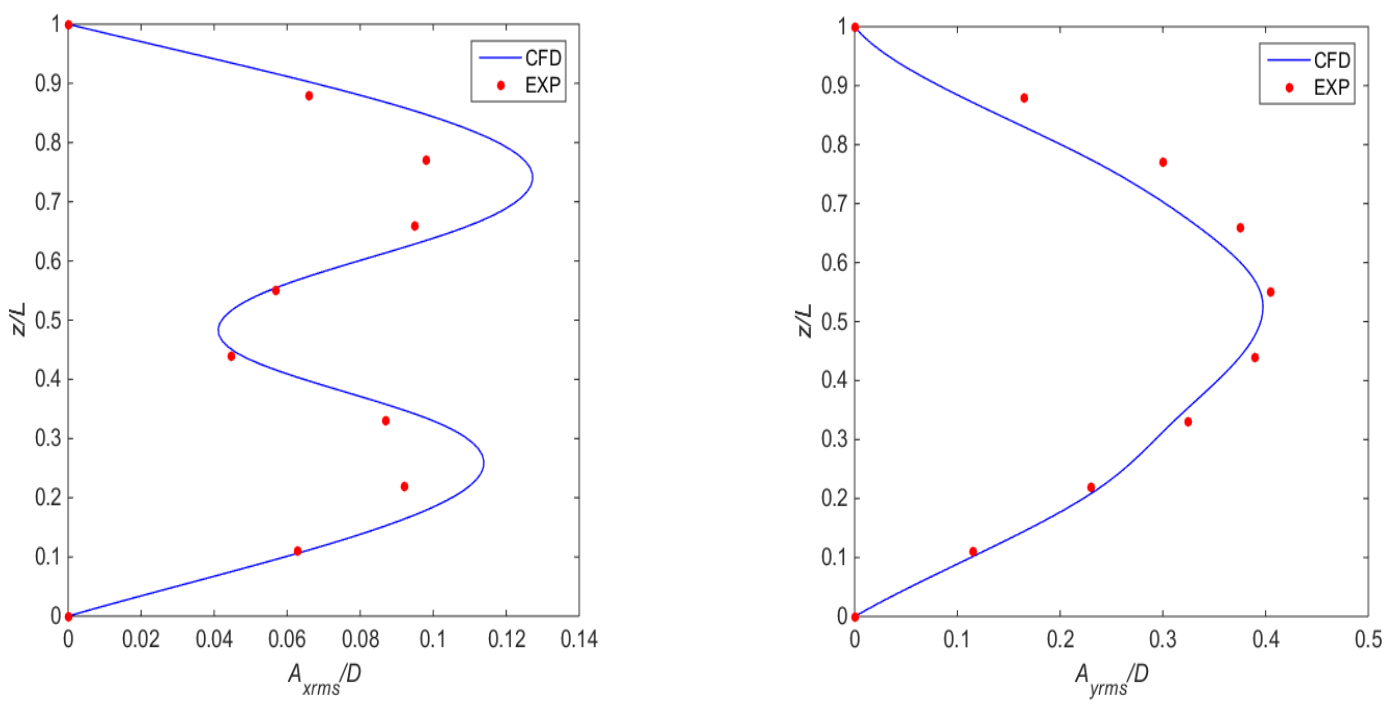

(a)
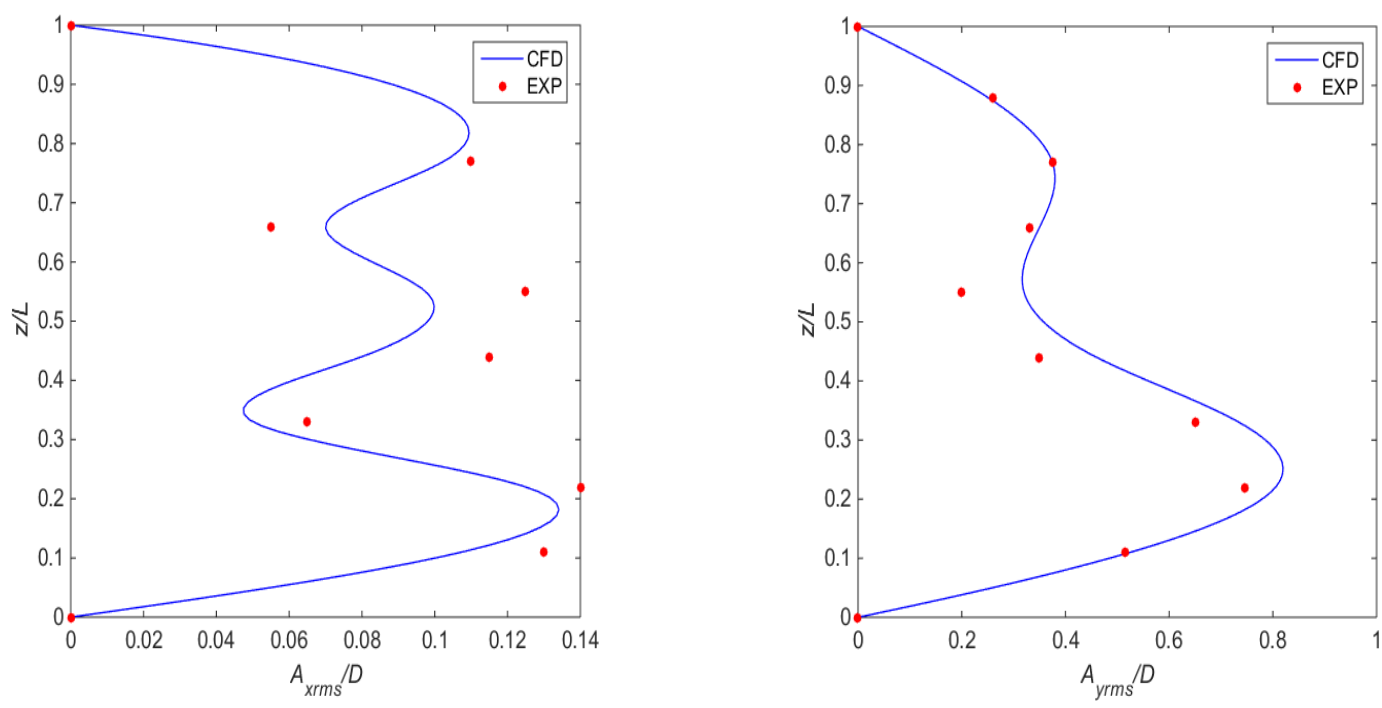

(b) 

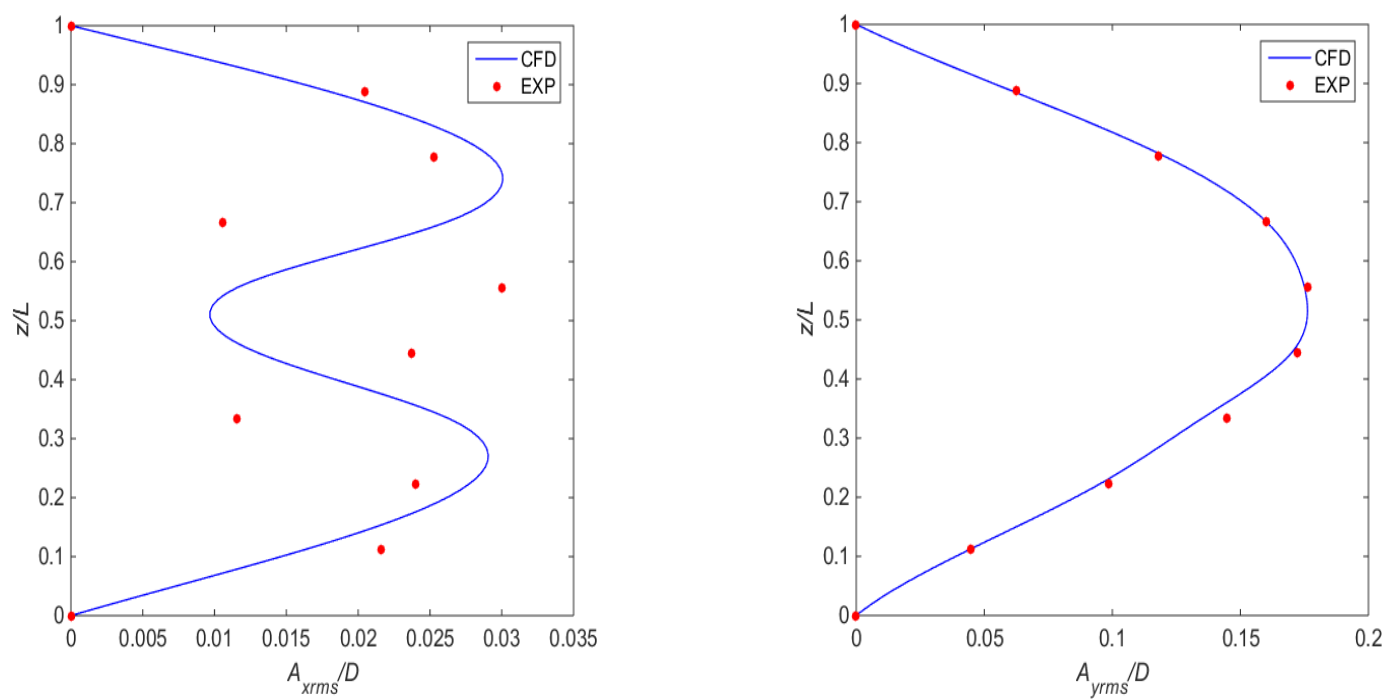

(c)
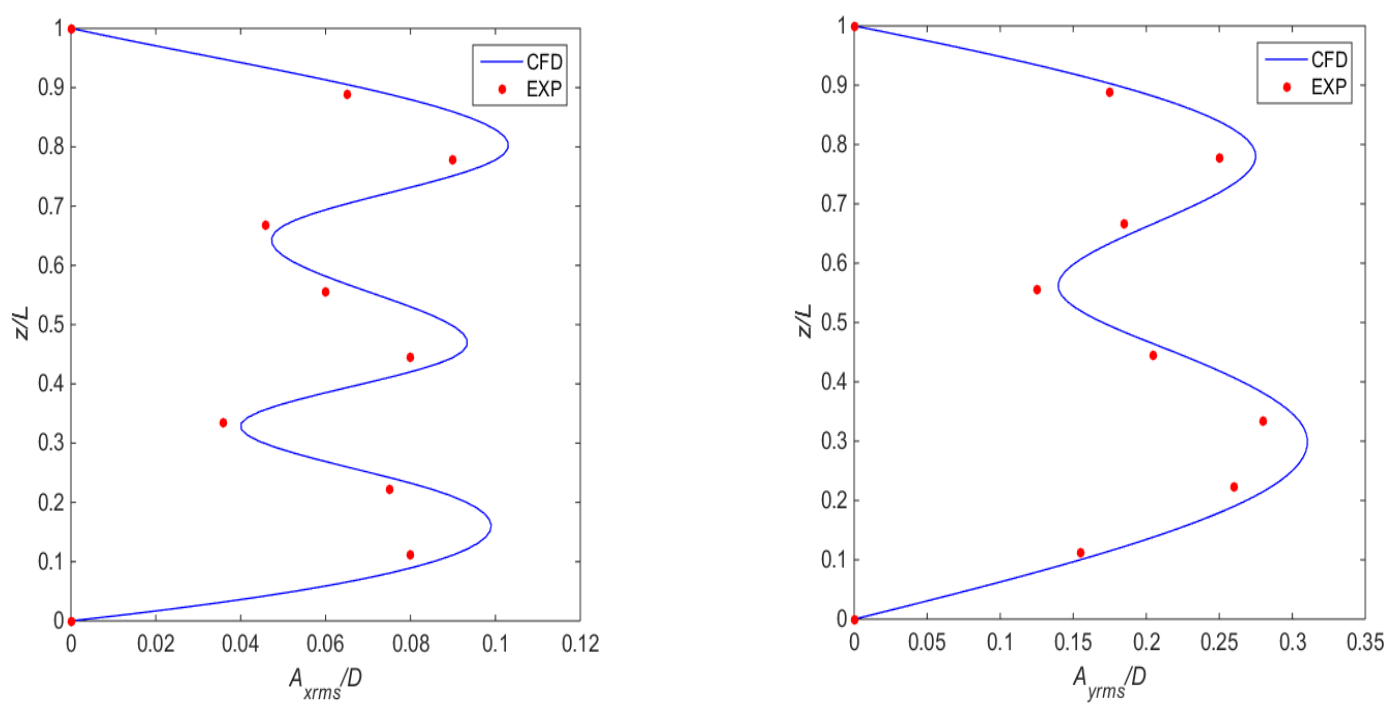

(d)

Fig. 8 Comparison of the rms amplitudes: (a) \# 1103, (b) \# 1105, (c) \# 1201 and (d) \# 1205. 

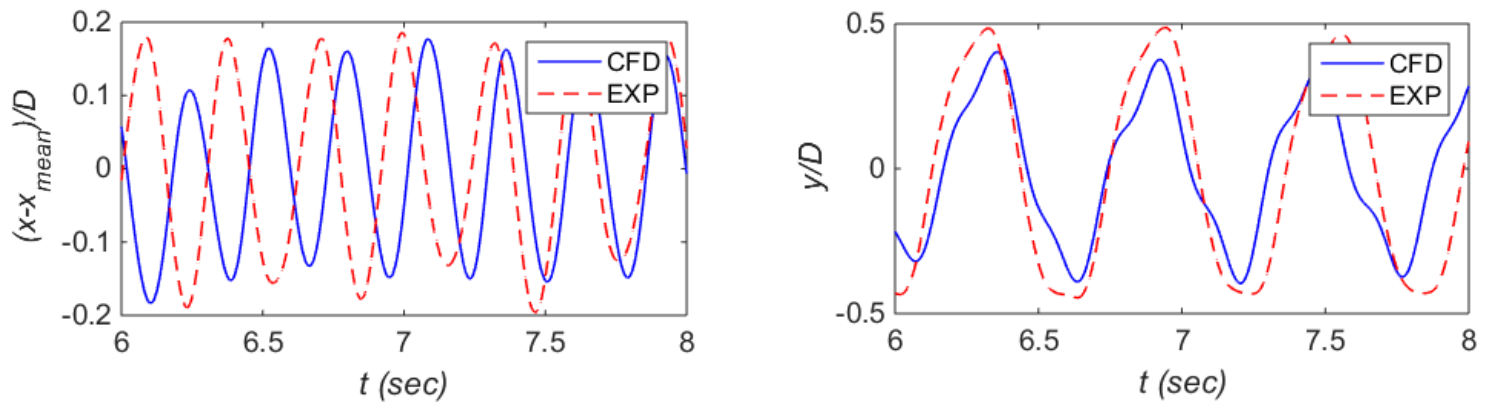

(a)
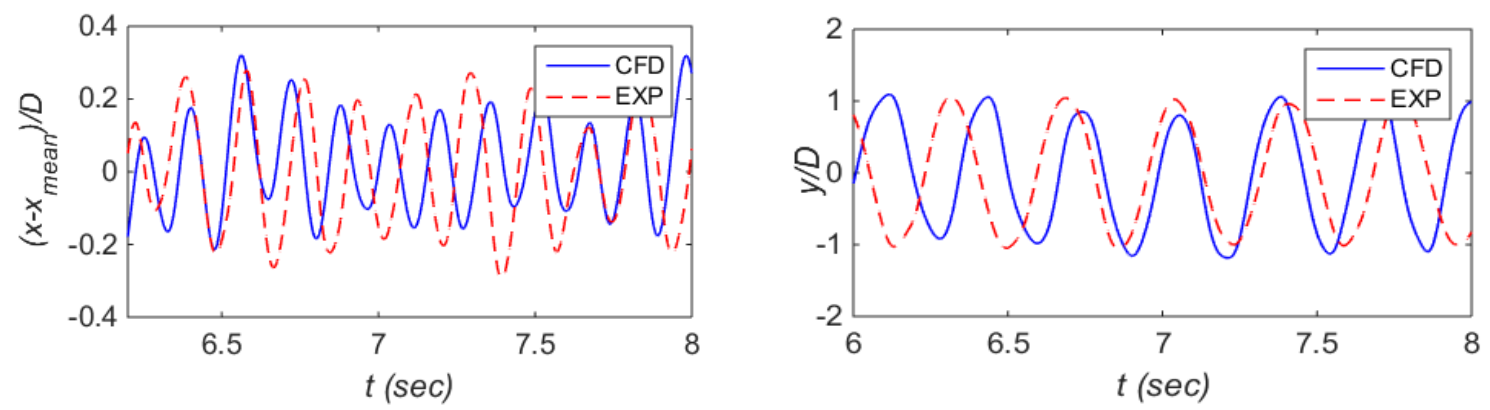

(b)
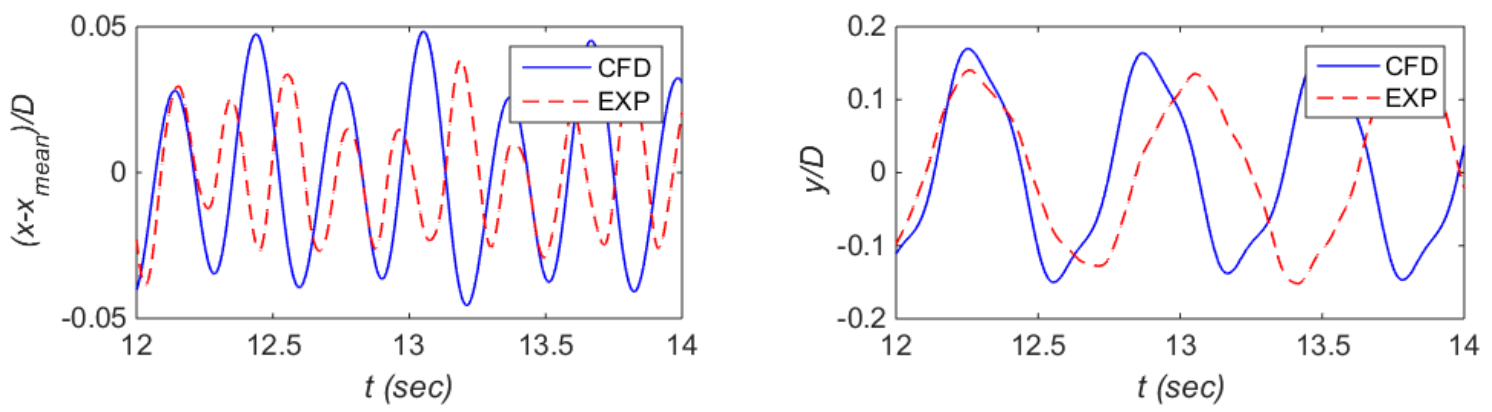

(c)
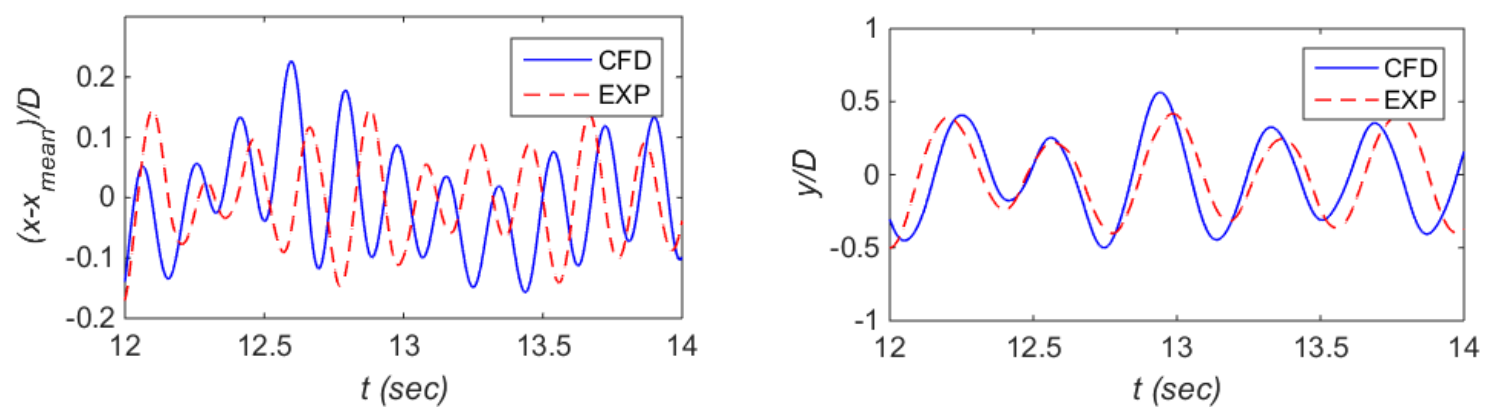

(d)

Fig. 9 Comparison of the displacement time histories at $z / L=0.22$ : (a) \# 1103, (b) \# 1105, (c) \# 1201 and (d) \# 1205. 
CFD

EXP

(a)
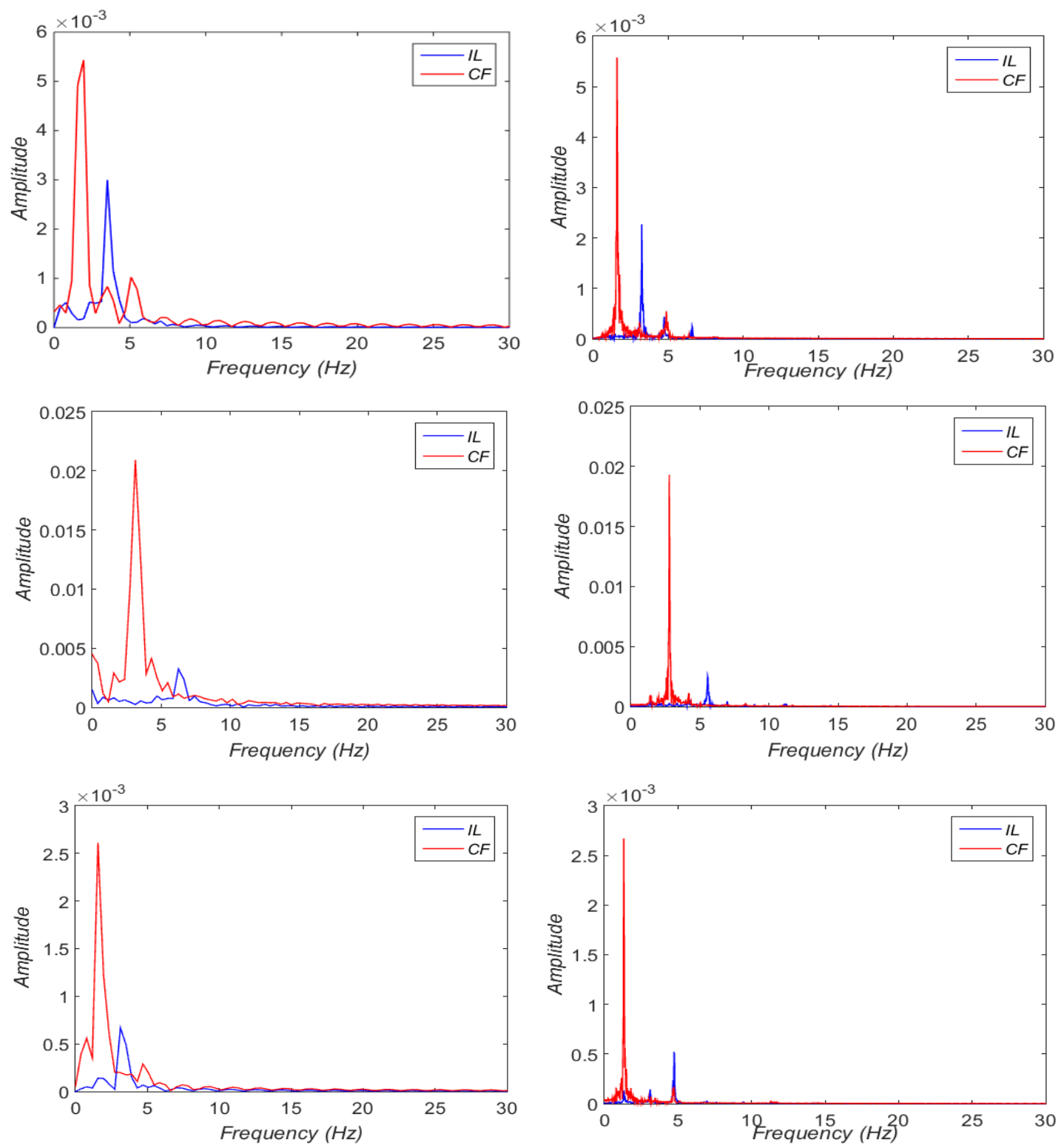

(c)
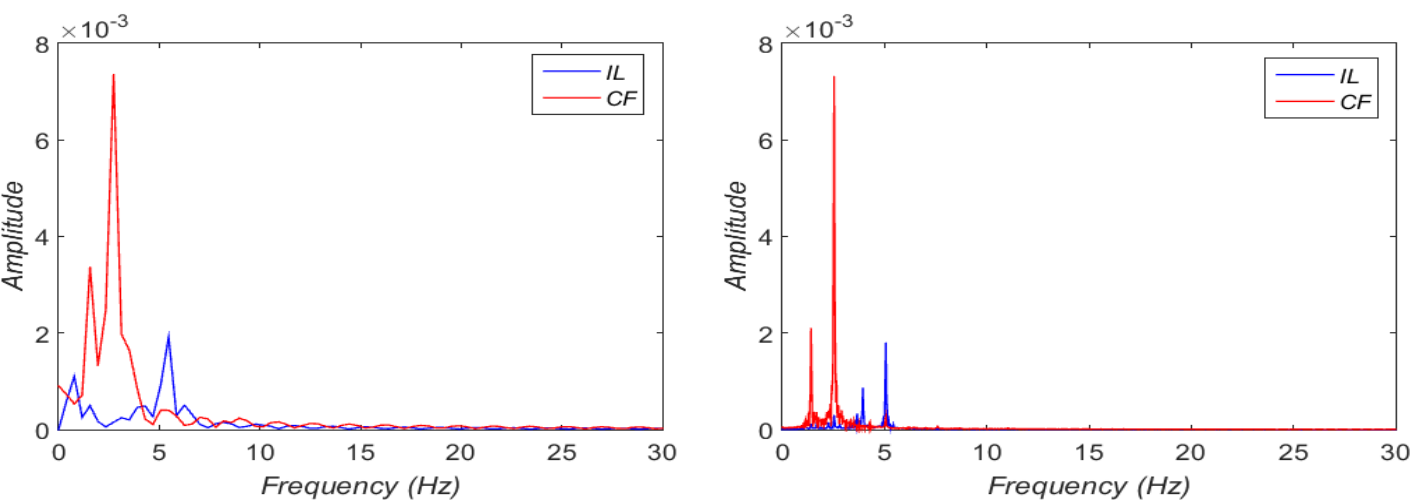

Fig. 10 Comparison of the oscillation frequencies at $z / L=0.22$ : (a) \# 1103, (b) \# 1105, (c) \# 1201 and (d) \# 1205. 
IL

$z / L=0.88$

$z / L=0.77$

$z / L=0.66$

$z / L=0.55$

$z / L=0.44$

$z / L=0.33$

$z / L=0.22$

$z / L=0.11$

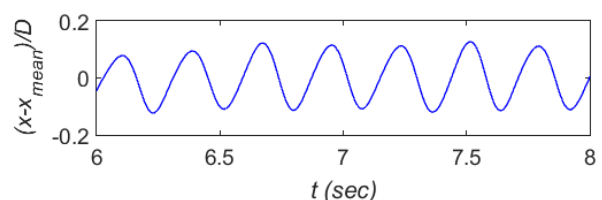

CF
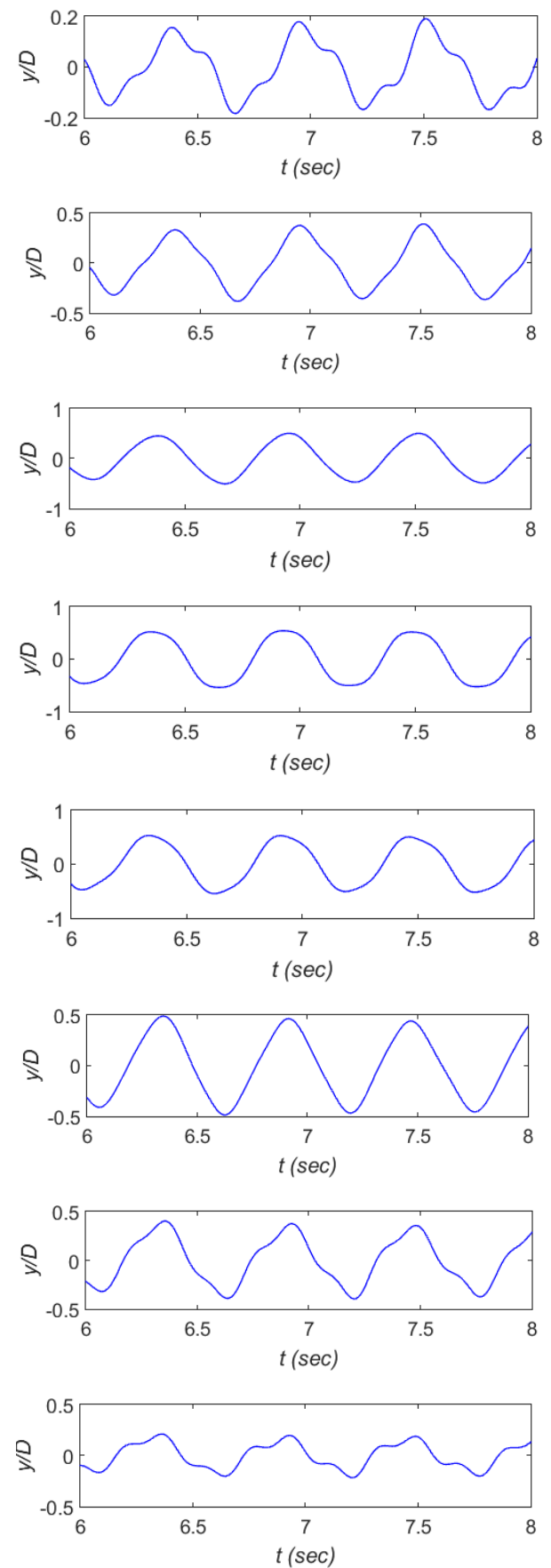

(a) 
IL

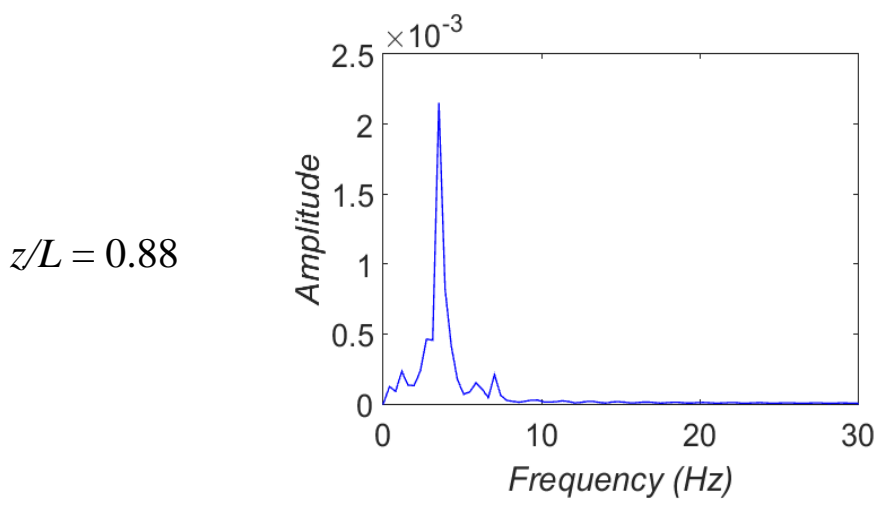

$z / L=0.77$

$z / L=0.66$
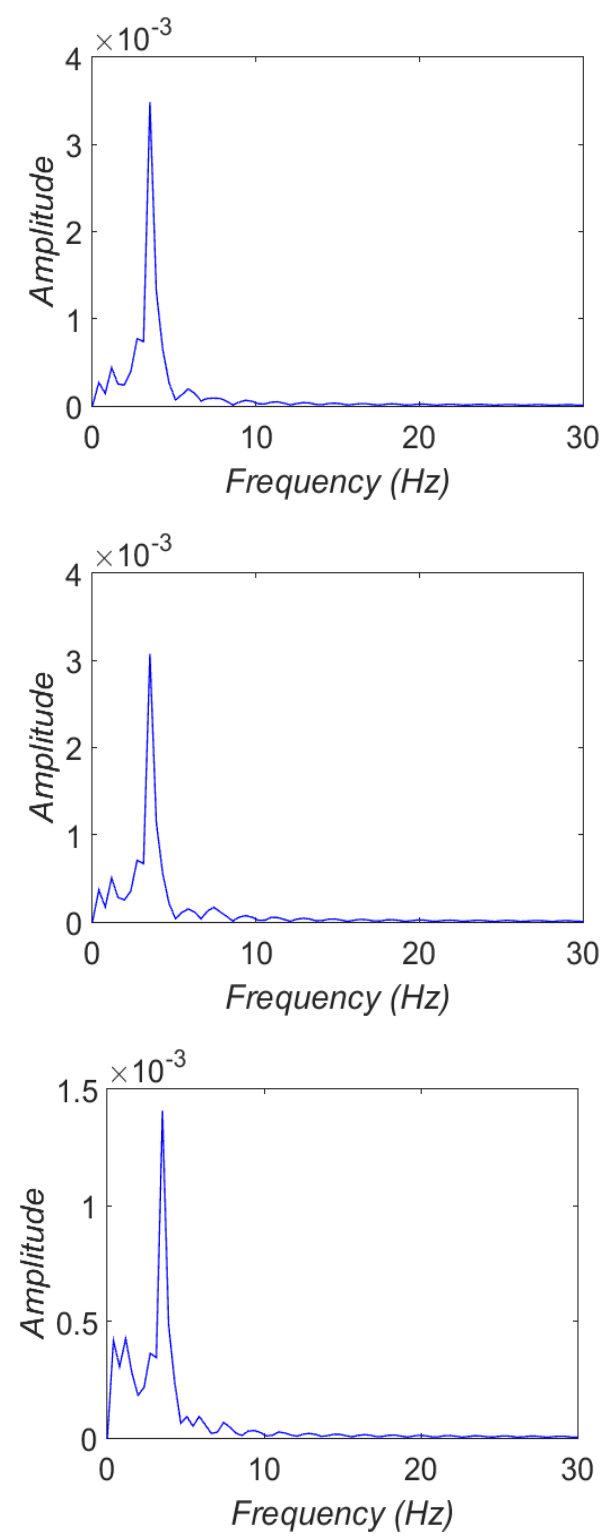

CF
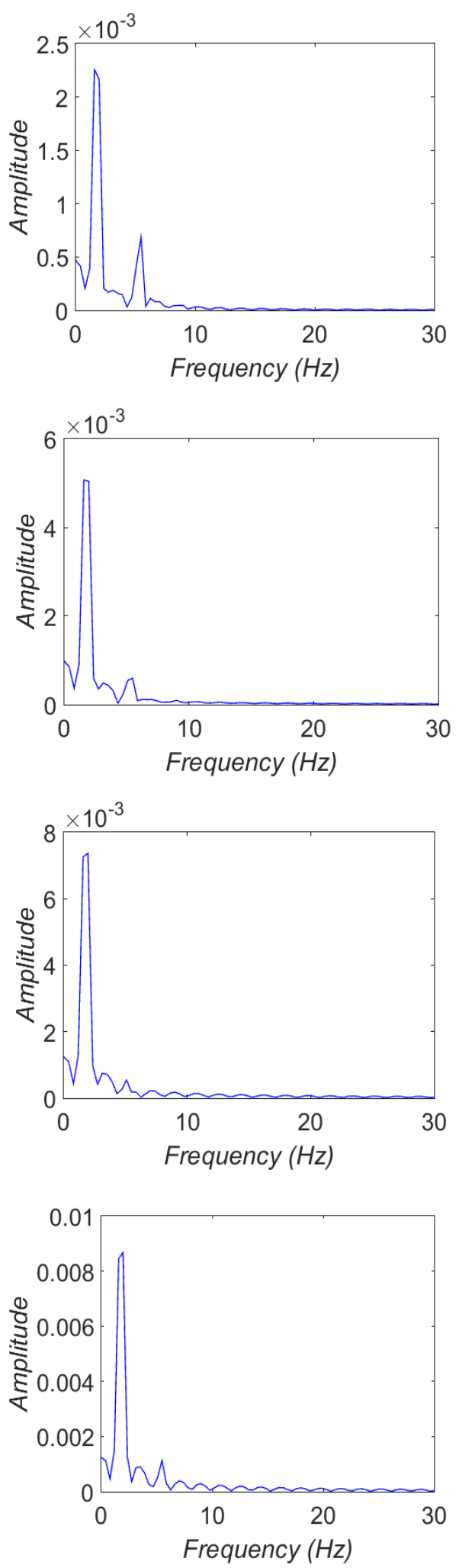

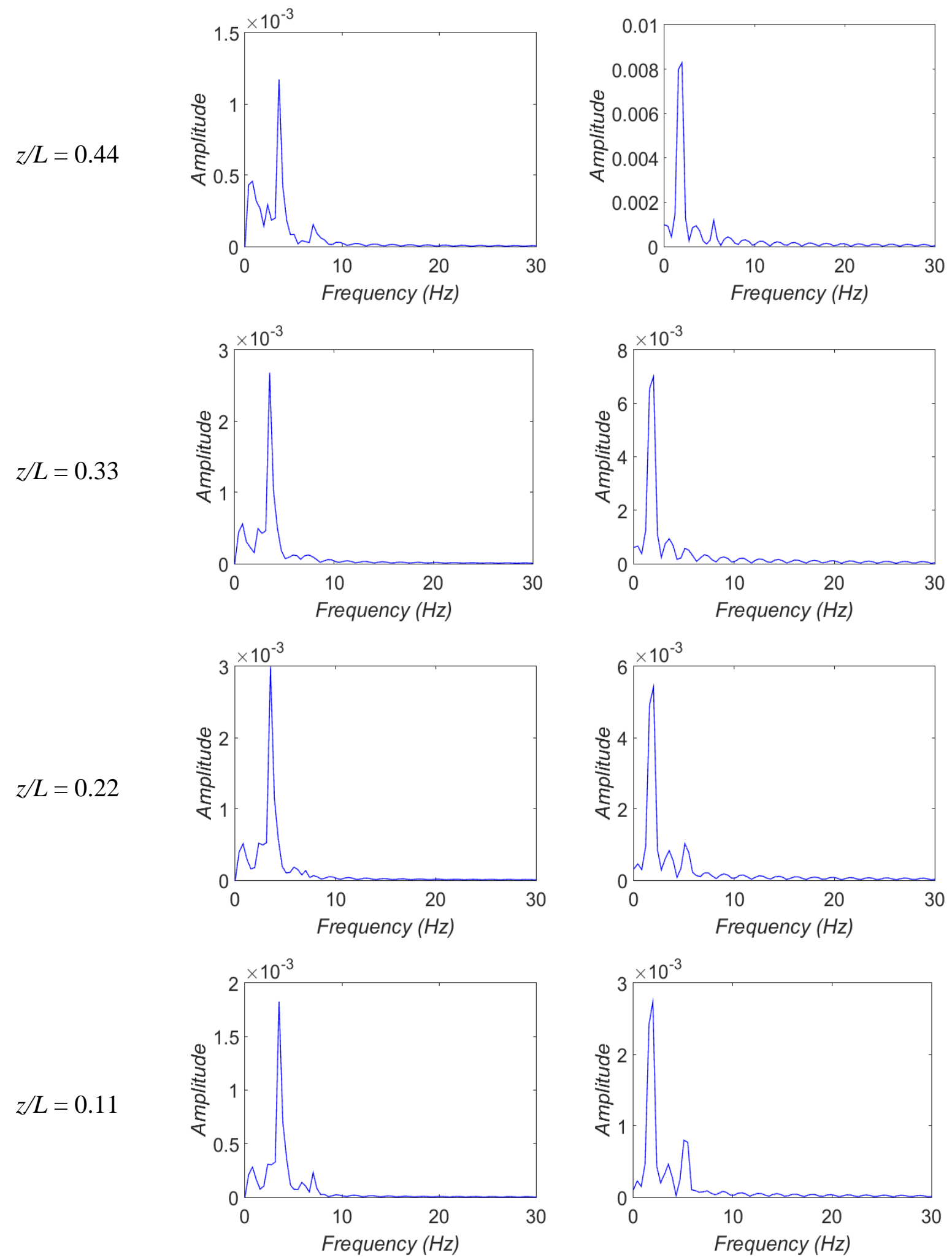

(b) 
IL

$z / L=0.88$

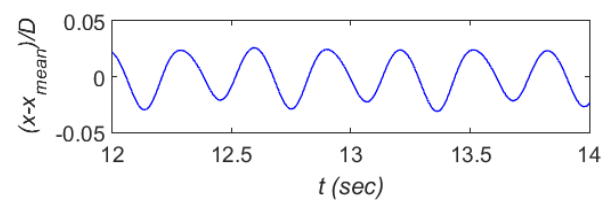

$z / L=0.77$
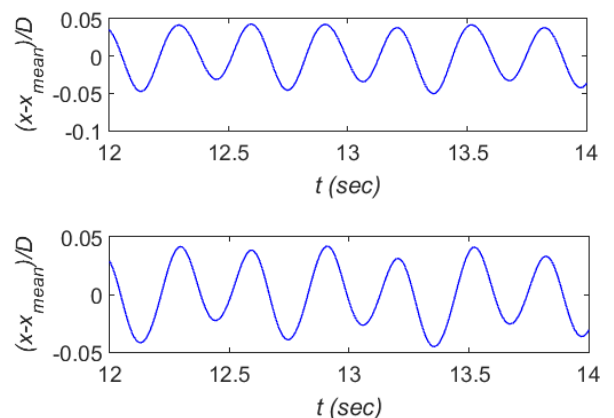

$z / L=0.66$

$z / L=0.55$

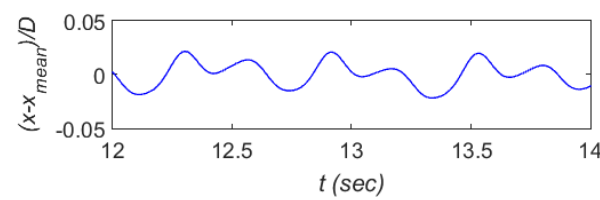

$z / L=0.44$

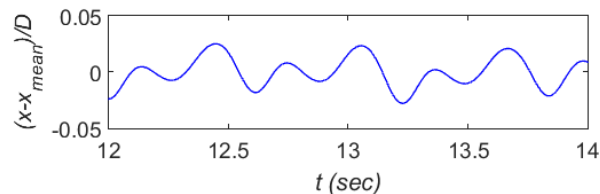

$z / L=0.33$

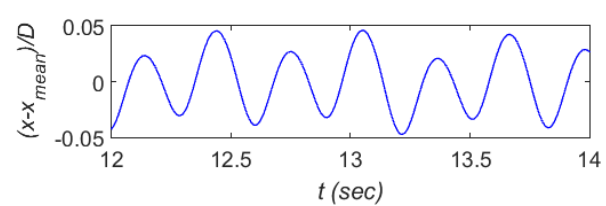

$z / L=0.22$

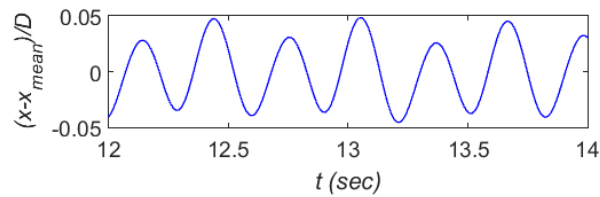

$z / L=0.11$
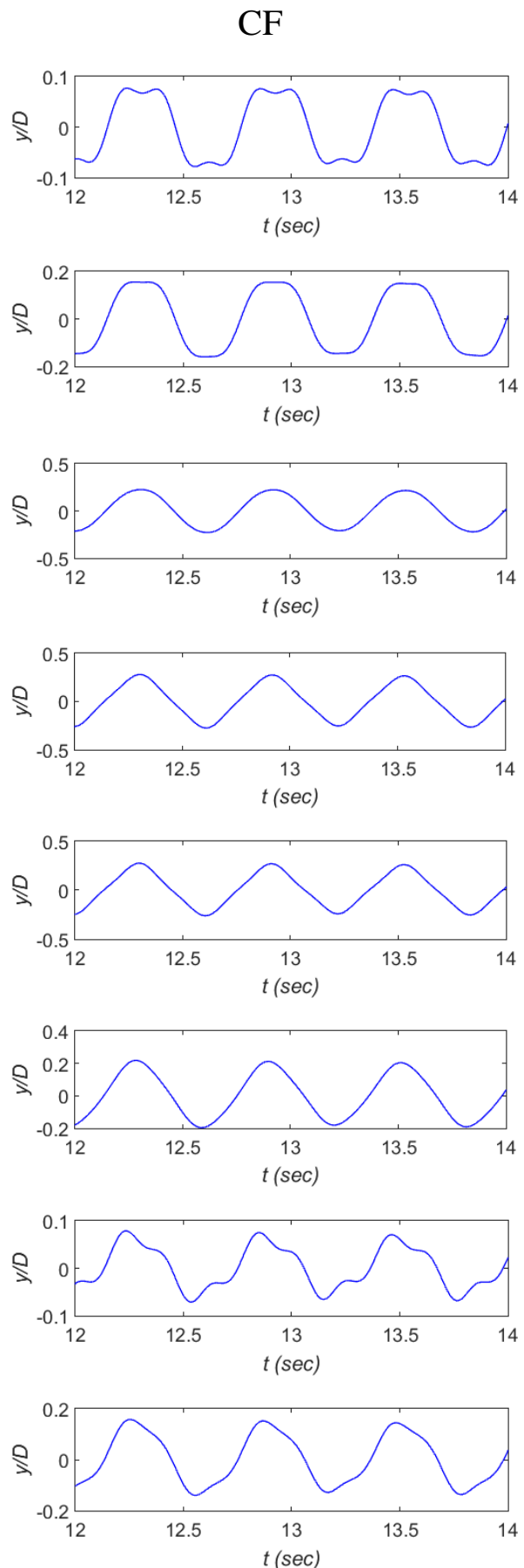

(c) 
IL
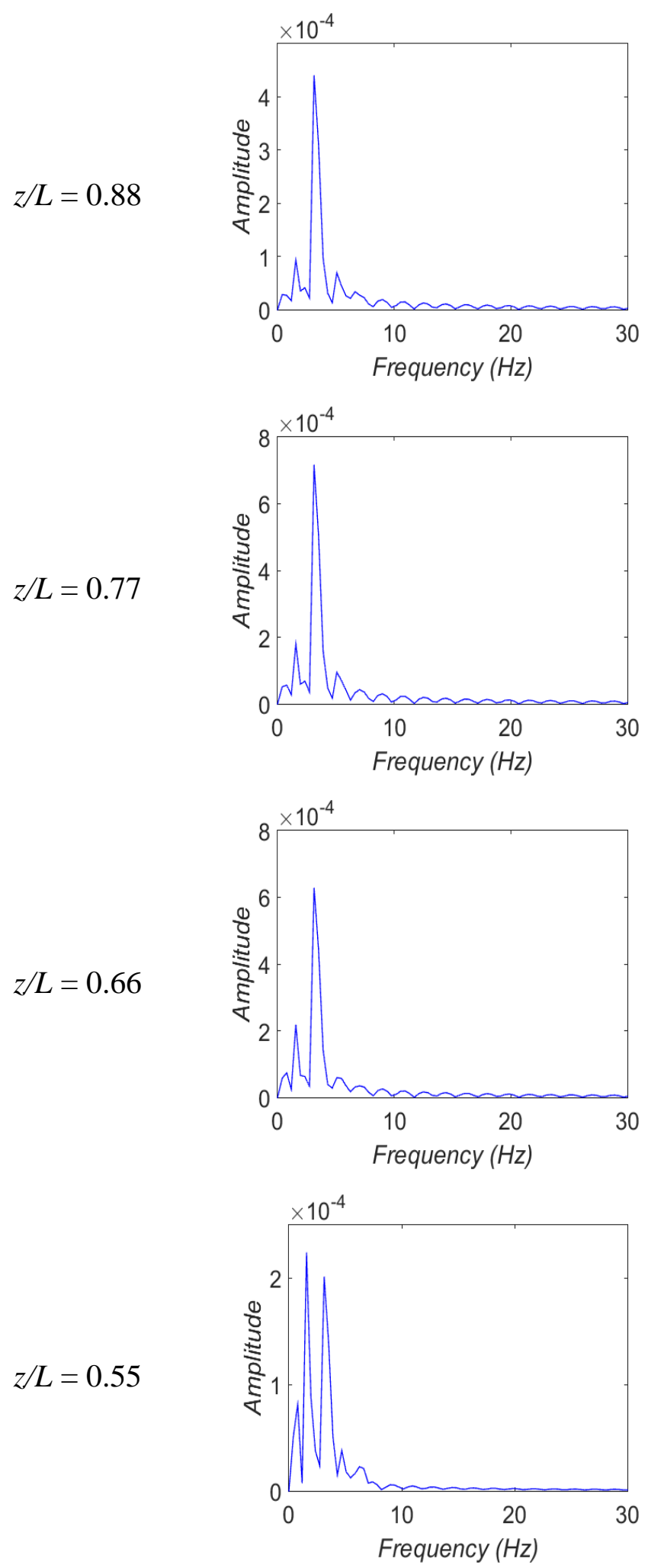

CF
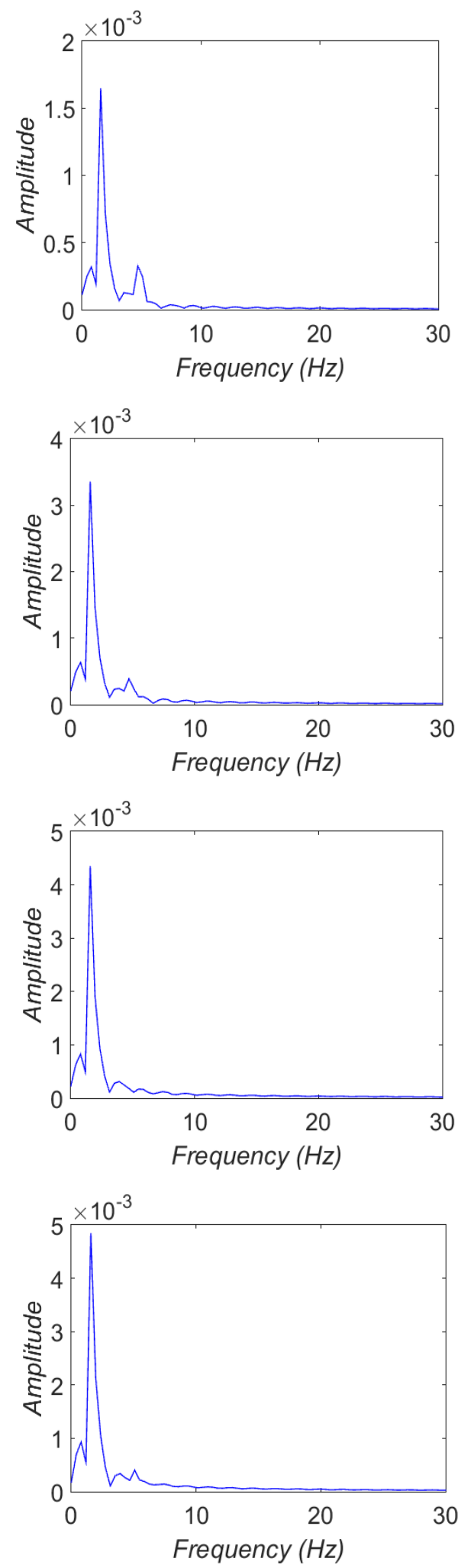

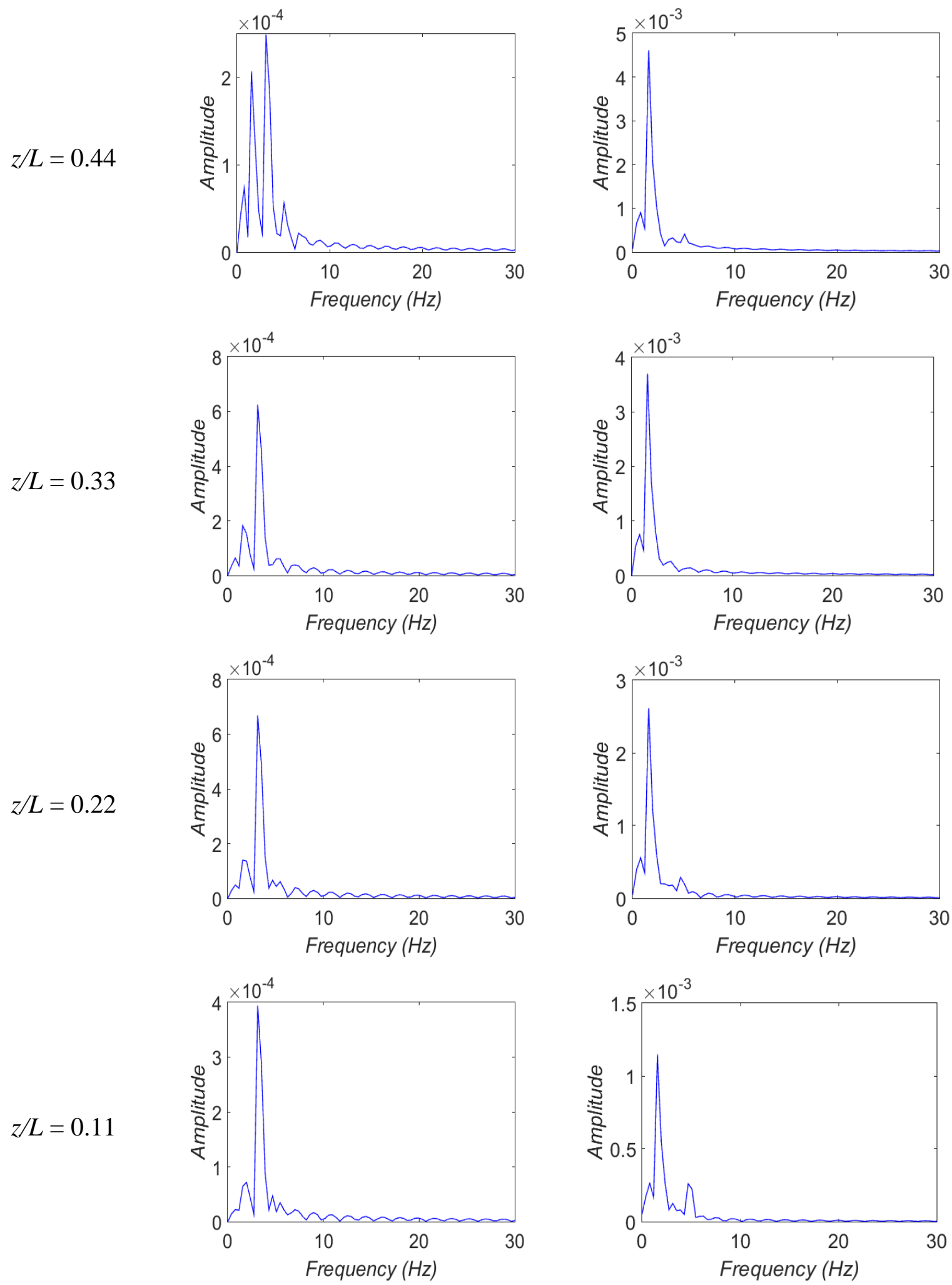

(d)

Fig. 11 Displacements and oscillation frequencies along the riser: (a) displacement time histories (\# 1103), (b) oscillation frequencies (\# 1103), (c) displacement time histories (\# 1201) and (d) oscillation frequencies (\#1201). 

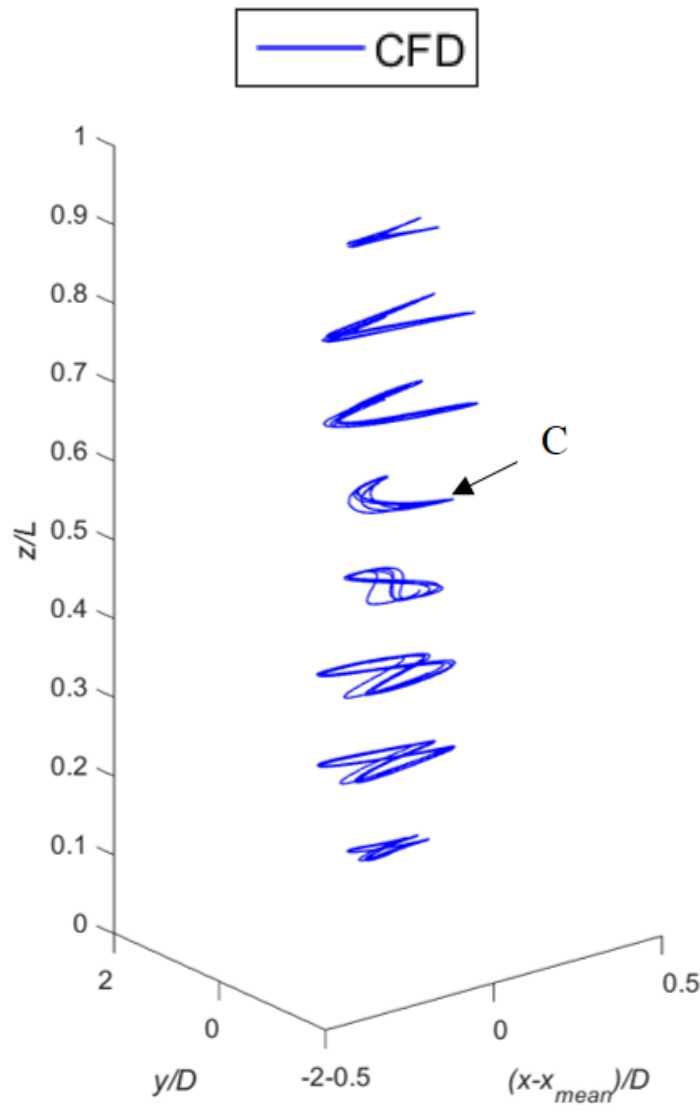

(a)
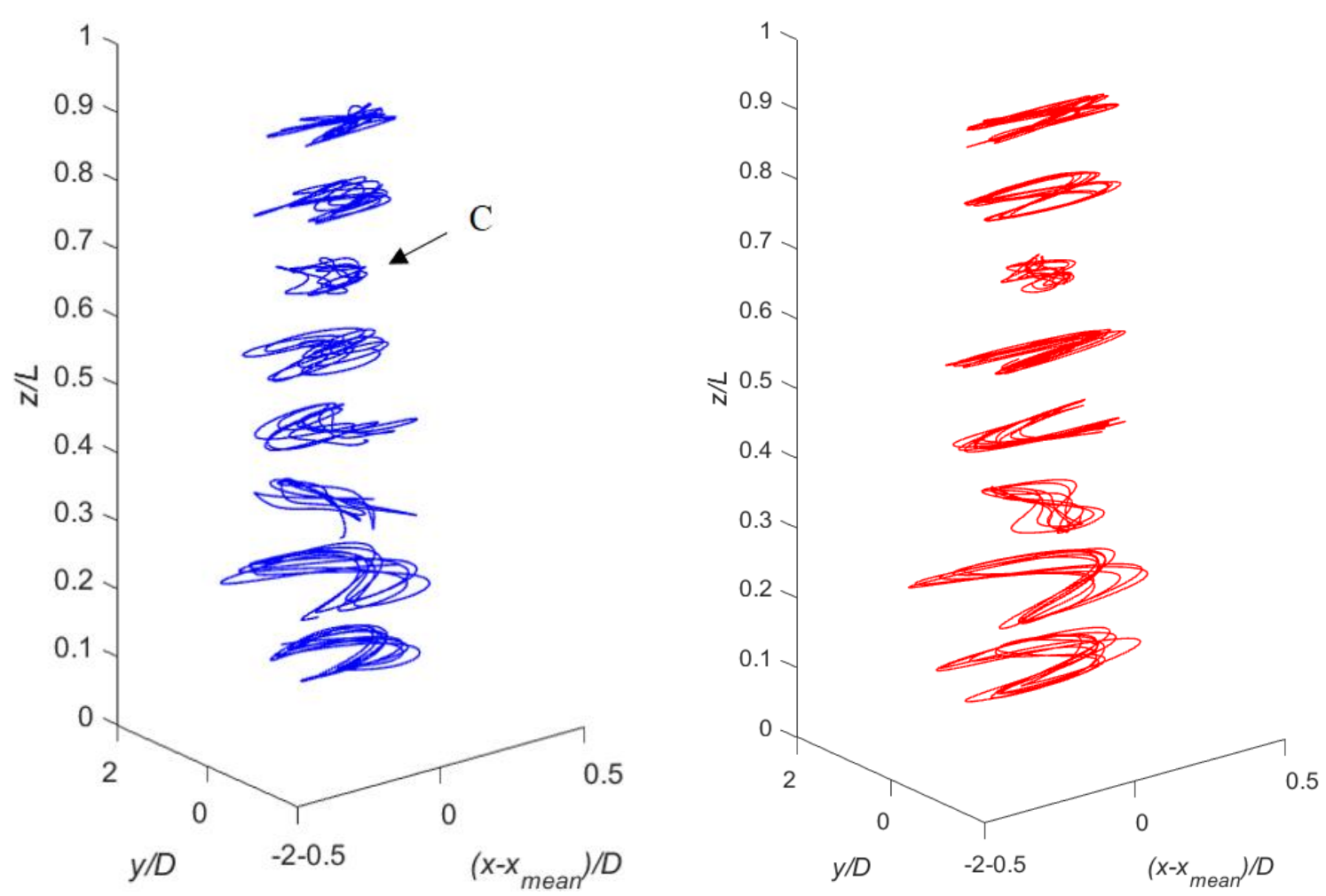

(b) 

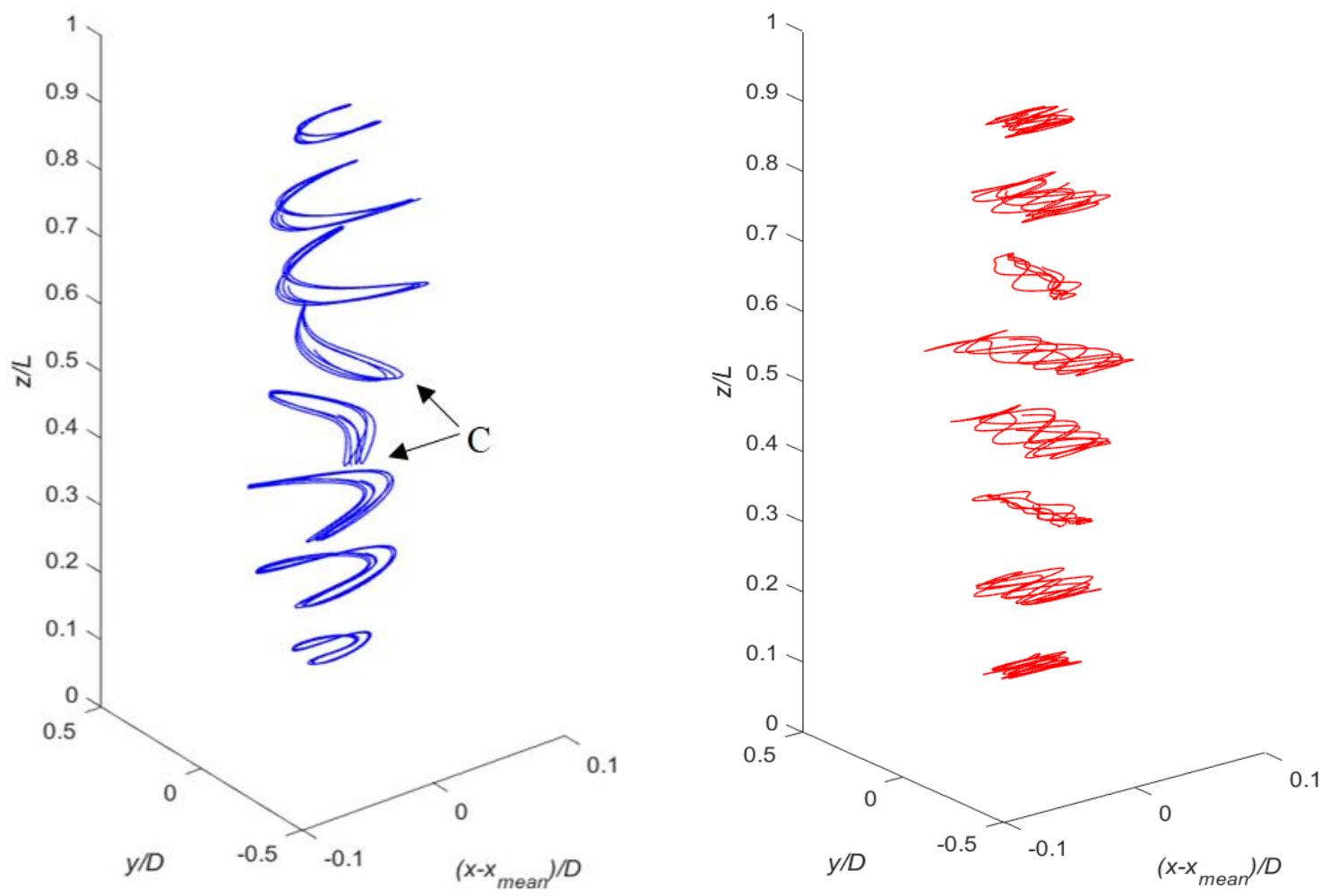

(c)
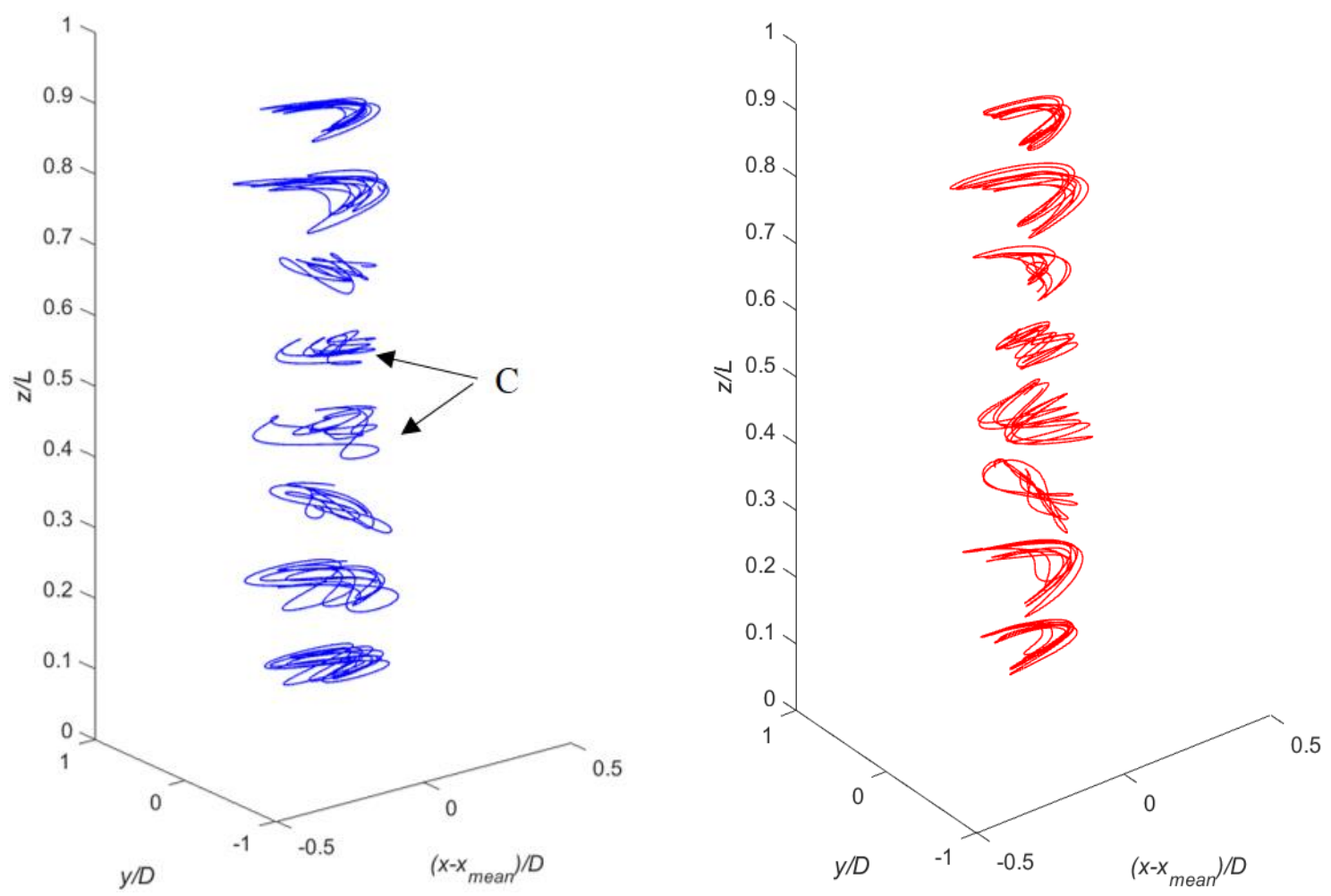

(d)

Fig. 12 Comparison of the orbital trajectories at various positions: (a) \# 1103, (b) \# 1105, (c) \# 1201 and (d) \# 1205. 


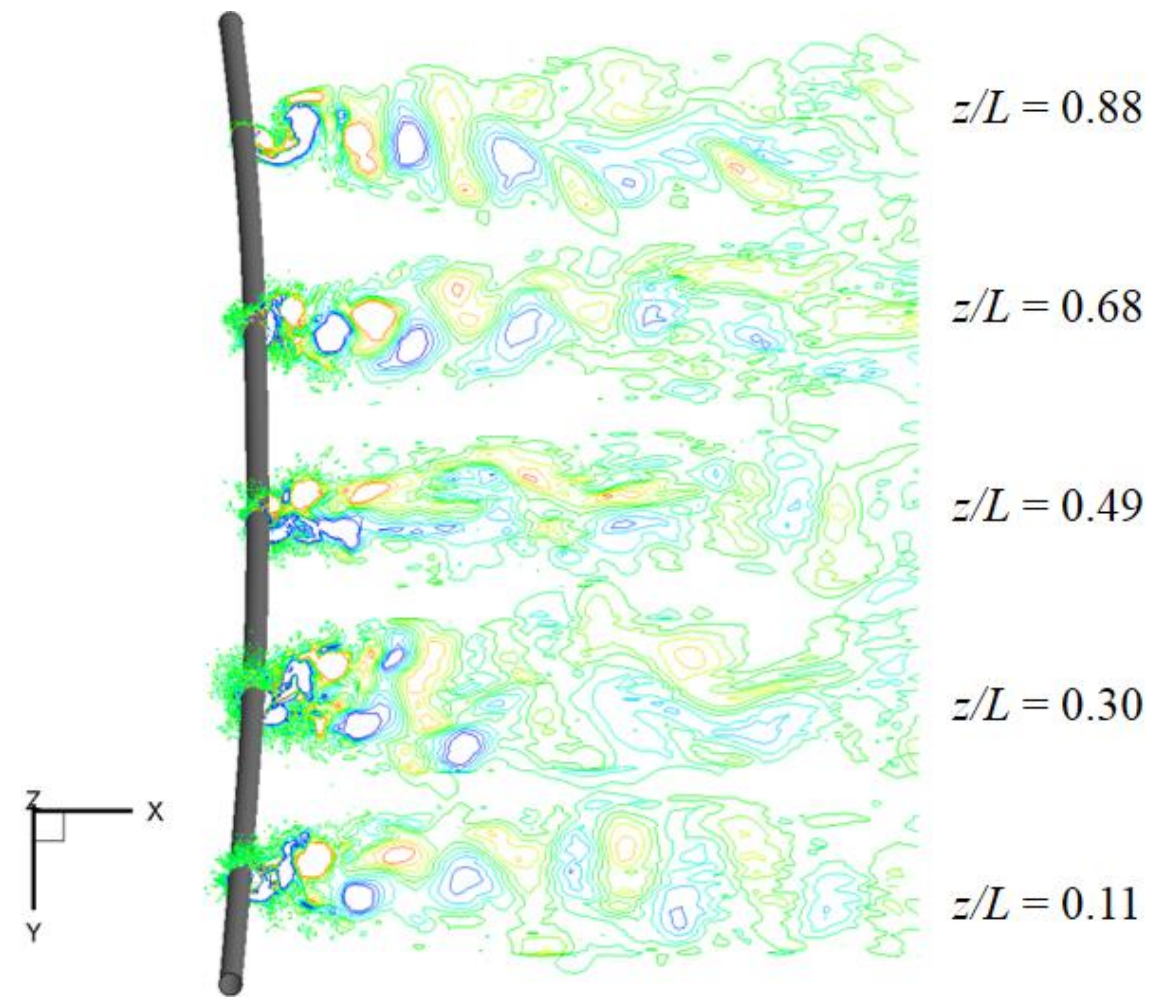

(a)

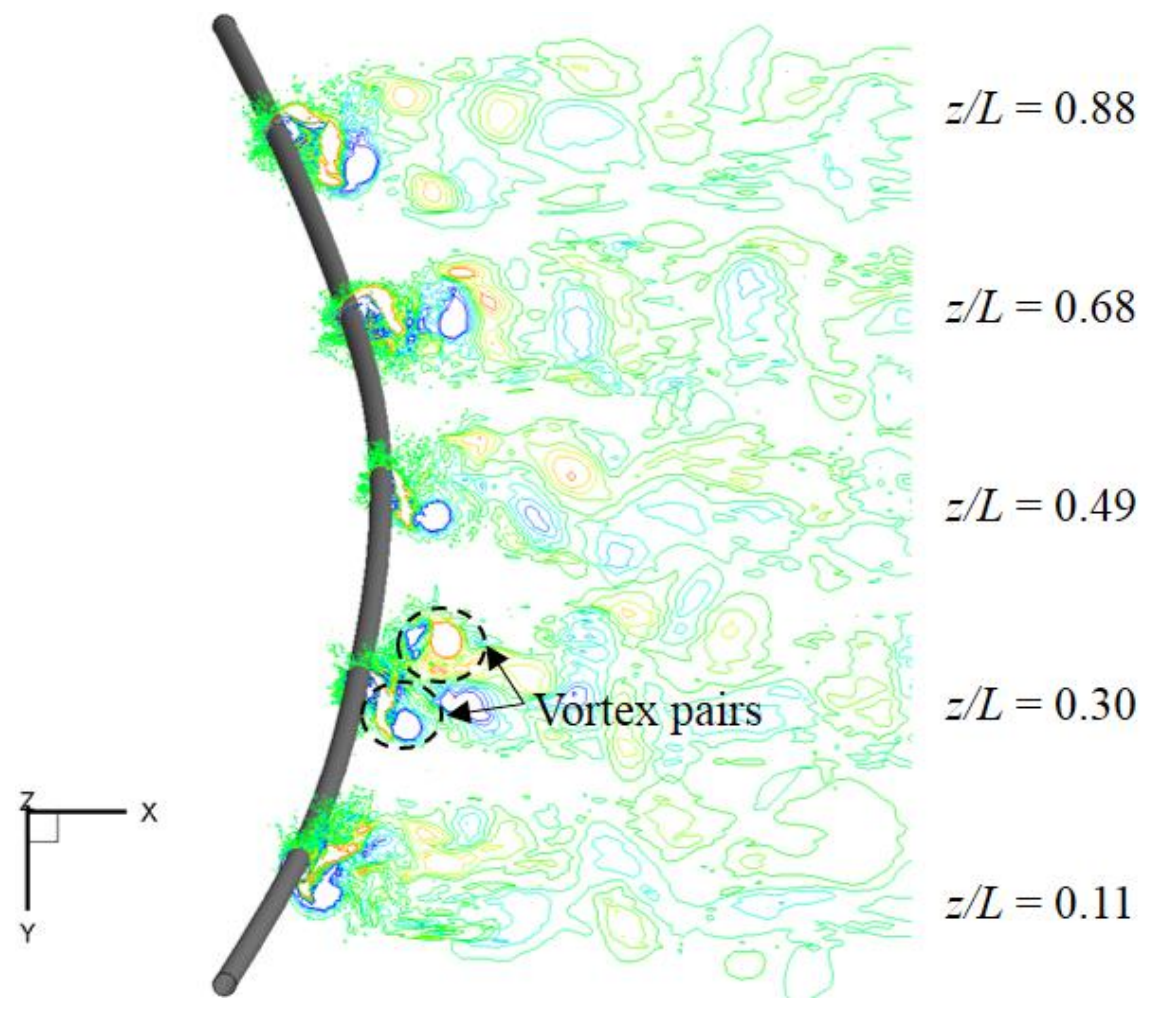

(b) 


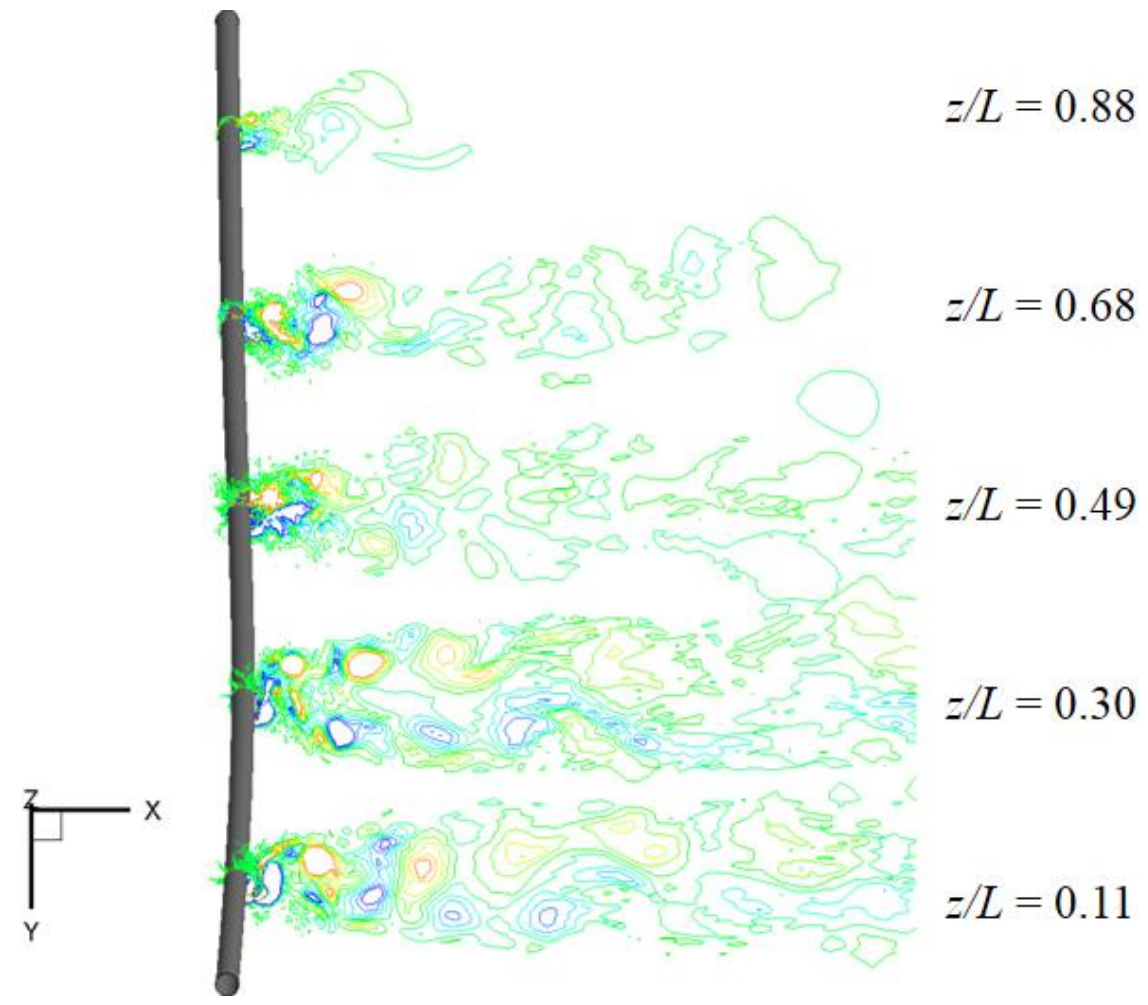

(c)

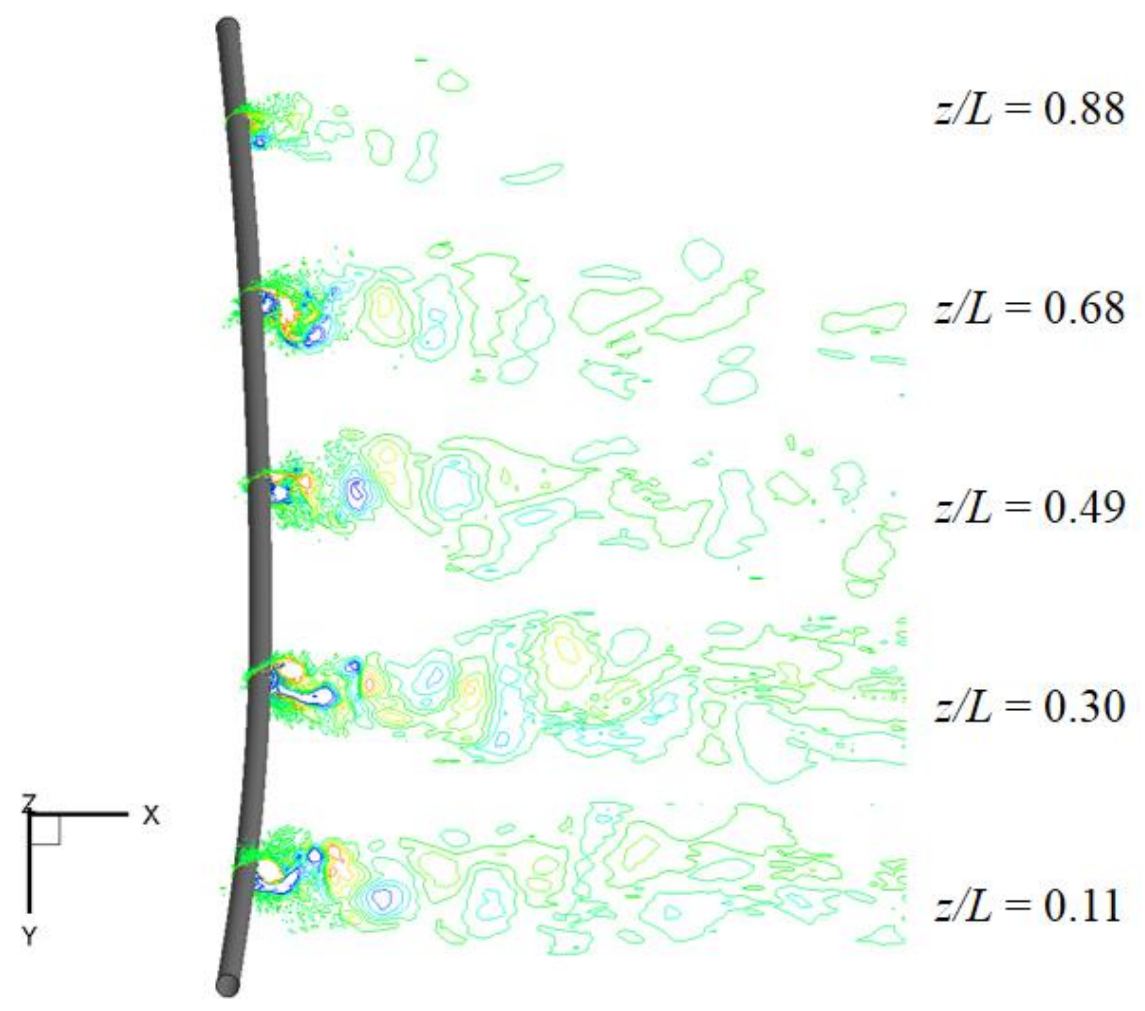

(d)

Fig. 13 Vortex shedding under different current profiles: (a) \# 1103, (b) \# 1105, (c) \# 1201 and (d) \# 1205. 

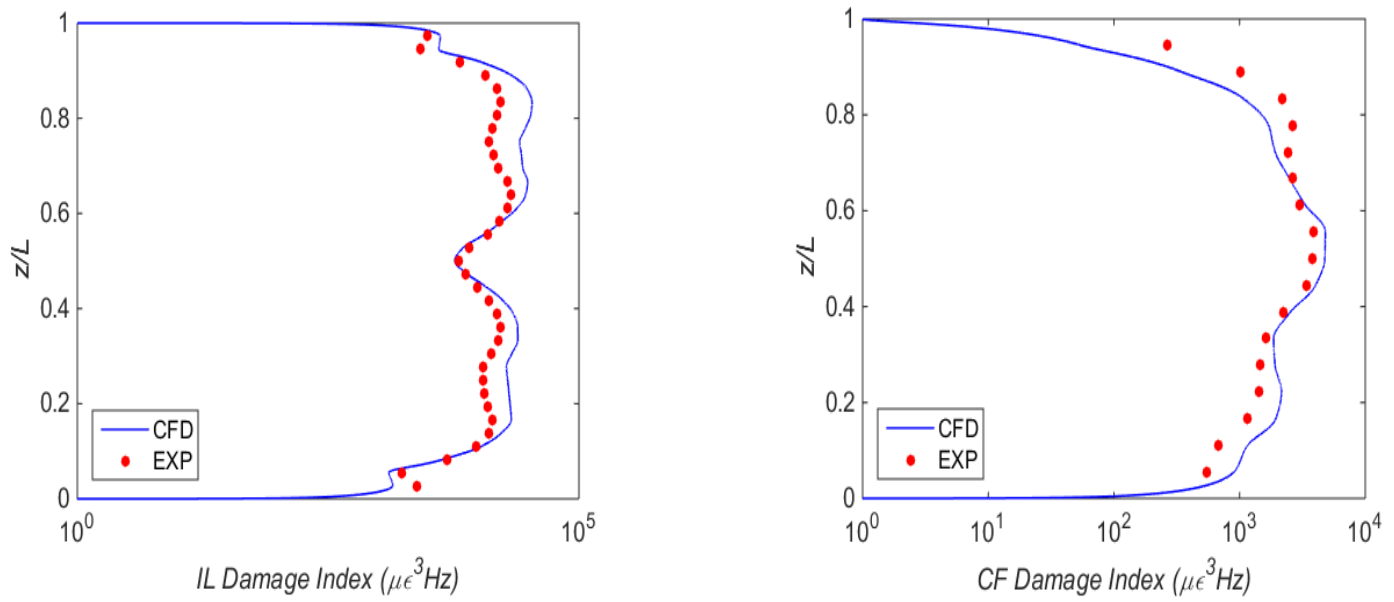

(a)
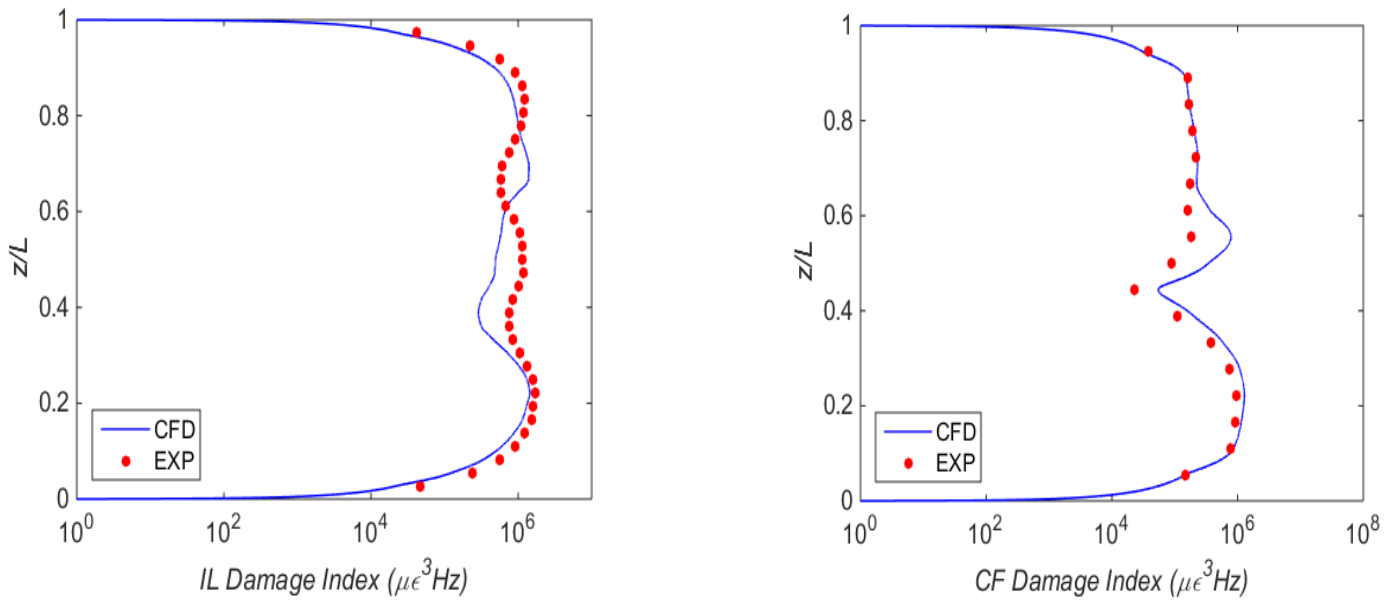

(b) 

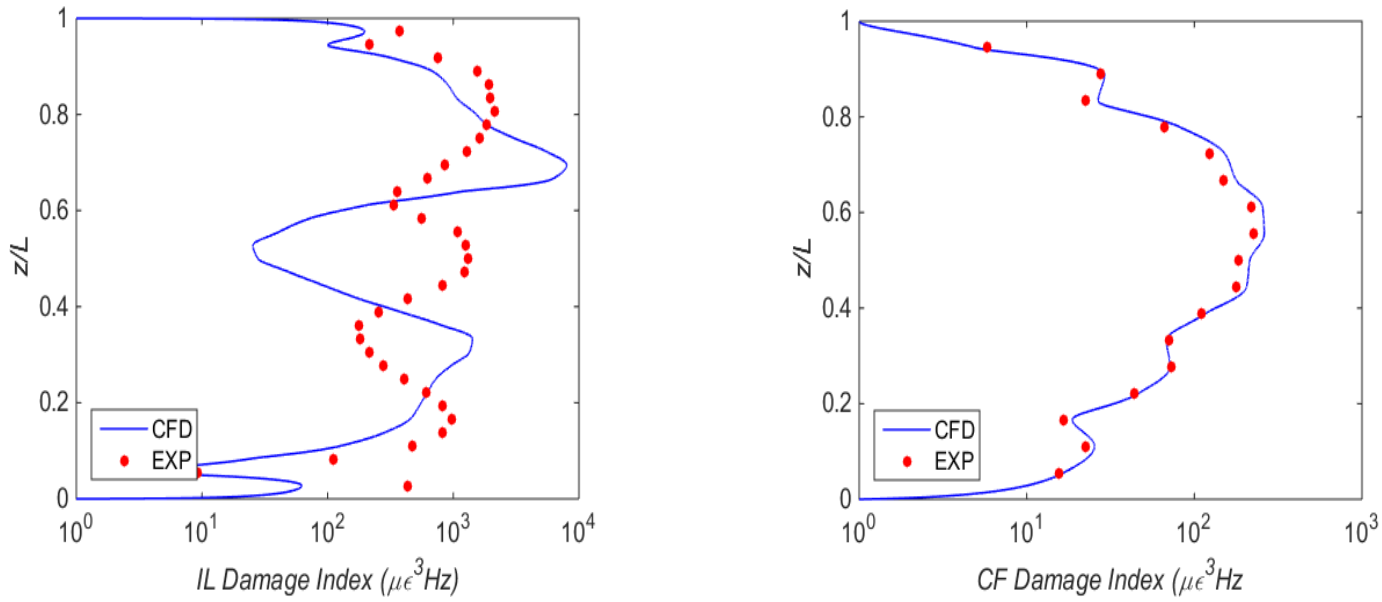

(c)
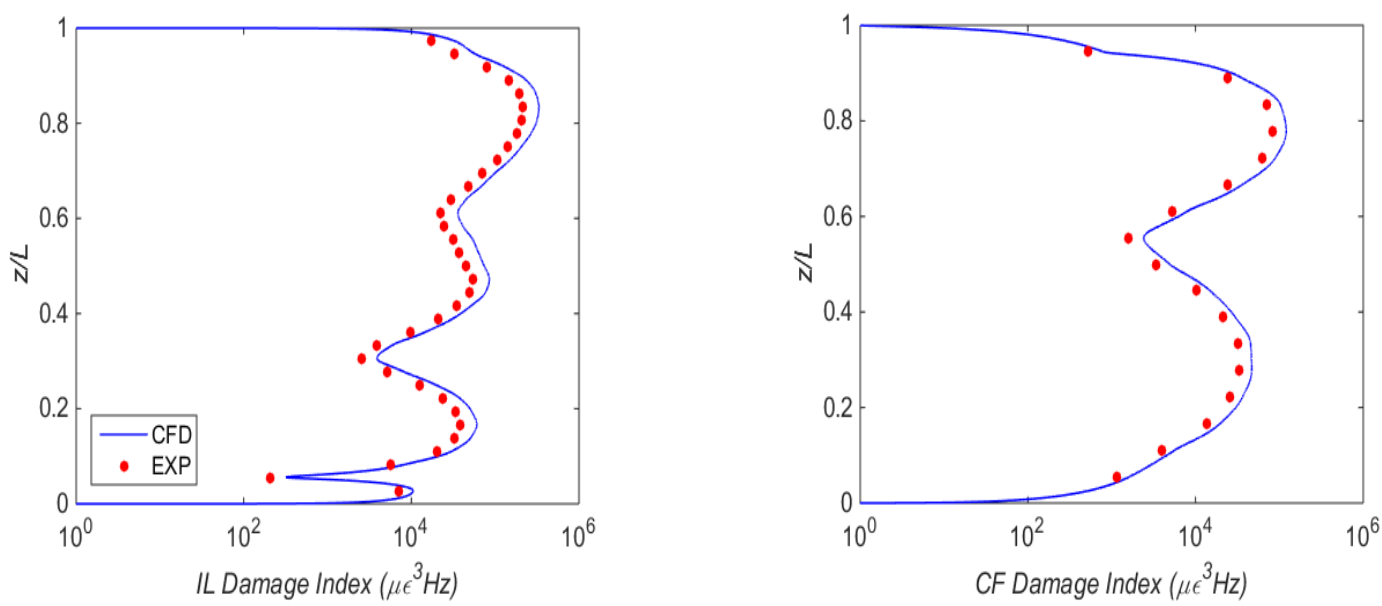

(d)

Fig. 14 Comparison of the fatigue damage indices: (a) \# 1103, (b) \# 1105, (c) \# 1201 and (d) \# 1205. 